\title{
Anomalous transport: a mathematical framework *
}

\author{
H. Schulz-Baldes ${ }^{\dagger}$, J. Bellissard ${ }^{\ddagger}$ \\ Université Paul-Sabatier, Toulouse, France ${ }^{\S}$
}

\begin{abstract}
We develop a mathematical framework allowing to study anomalous transport in homogeneous solids. The main tools characterizing the anomalous transport properties are spectral and diffusion exponents associated to the covariant Hamiltonians describing these media. The diffusion exponents characterize the spectral measures entering in Kubo's formula for the conductivity and hence lead to anomalies in Drude's formula. We give several formulas allowing to calculate these exponents and treat, as an example, Wegner's $n$-orbital model as well as the Anderson model in coherent potential approximation.
\end{abstract}

\section{Introduction}

\subsection{Anomalous electronic transport}

Quantum effects and interactions in various materials cause a great variety of behaviors for electronic transport at low temperature. Understanding why some materials are conductors and others insulators is a challenging central problem of solid state physics. The first attempt to get a microscopic theory of electronic transport goes back to the work of Drude [27] who wrote the conductivity in the form:

$$
\sigma=\frac{n q^{2} \tau}{m_{*}}
$$

where $n$ is the charge carrier density, $q$ is the carrier electric charge, $m_{*}$ is the carrier effective mass and $\tau$ is the collision time. The derivation of this formula was initially given in terms of kinetic transport of classical particles, but Sommerfeld and Peierls rederived it in the context of quantum theory of crystals which led to consequences in better agreement with experiments in metallic samples [5]. The main weakness of the theory lies in the definition of $\tau$. The collision time is often understood as a phenomenological parameter that can be easily measured, but is difficult to interpret. This is the so-called relaxation time approximation (RTA). However, using more sophisticated theories, one can calculate it if various contributions such as electronimpurity, electron-phonon or electron-electron scattering are taken into account. It gives a

*to appear in Reviews in Mathematical Physics

$\dagger$ †e-mail: hermann@irsamc2.ups-tlse.fr

${ }^{\ddagger} \mathrm{e}-\mathrm{mail}$ : jeanbel@irsamc2.ups-tlse.fr

$\S$ UMR 5626, CNRS and Laboratoire de Physique Quantique, 118, Route de Narbonne, 31062-Toulouse Cedex, France 
temperature dependence in the form of a power law, namely $\tau(T) \sim T^{\gamma}$, where $\gamma$ depends upon the type of collision which dominates dissipation [5].

Among the various mechanisms that may lead to a metal-insulator transition, one is the anomalous quantum transport. This means that, within a one-electron effective theory, the electronic wave packet diffuses anomalously through the systems instead of moving ballistically between collisions as free electrons in a perfect crystal. Such a mechanism is probably at the basis of the strange transport properties of quasicrystals at low temperature [17, 50]. The best samples are nowadays alloys made of very good metals, such as the $A l_{62.5} C u_{25} F e_{12.5}$, $A l_{70.5} P d_{22} M_{7.5}$ or $A l_{70.5} P d_{21} R e_{8.5}$, crystallizing in a quasiperiodic icosahedral phase. They have conductivities comparable to doped semiconductors, namely $10^{-6}$ to $10^{-9}$ times less than for pure aluminum at $4 K$ (see for instance [56, 64] for the following informations); moreover, the conductivity increases with temperature, which is just the opposite of the behavior of a normal metal; the temperature dependence of the conductivity is neither of the exponential type $\exp \left(-E / k_{B} T\right)$ characterizing thermally activated processes, nor of the form $\exp \left(-a T^{-\alpha}\right)$ as in Mott's hopping conductivity or related mechanisms [68], but rather exhibits a power law behavior $\sigma(T) \sim T^{\beta}$ in the range $4-800 K$ with $1<\beta<1.5$ [64]; at last, the behavior of the conductivity as a function of the magnetic field shows the typical weak localization signature observed in slightly disordered metals [64].

On the other hand, several numerical results concerning the time behavior of tight binding models on a quasiperiodic lattice have shown that the spreading of the wave packet satisfies a power law behavior in time of the type [38, 29, 73]

$$
\left\langle\phi\left|(\vec{X}(t)-\vec{X}(0))^{2}\right| \phi\right\rangle \sim t^{2 \sigma_{\text {diff }}} \quad \text { as } \quad t \rightarrow+\infty,
$$

where $|\phi\rangle$ is the initial localized wave function and $\vec{X}$ is the position operator for the particle. Depending on the strength of the quasiperiodic potential, the diffusion exponent $\sigma_{\text {diff }}$ may vary from 0 to 0.8 in a nearest neighbor model on an octagonal lattice [73]. Similar behavior were observed in the Harper model [38, 29, the Fibonacci Hamiltonian 38] and the kicked Harper model [3]. For quasicrystals, analytical calculations in one dimension [55] or more concrete phenomenological models in three dimension [42 have led to similar results and to predictions for the diffusion exponent $\sigma_{\text {diff }}$.

Experimental results, their theoretical interpretation and numerical simulations have therefore led the experts toward the idea that anomalous diffusion as described in equation (2) is the main reason for the strange transport properties of quasicrystals, at least for temperatures above approximately $4 K$ (below $4 K$, electron-electron interactions may become more important). One consequence of (2), as we will show in this paper, is that the conductivity in RTA no longer satisfies the Drude formula (1), but rather:

$$
\sigma \sim \tau^{2 \sigma_{\mathrm{diff}}-1} \quad \text { as } \quad \tau \rightarrow+\infty
$$

Such a formula can be guessed by means of the following non rigorous argument [72, 50]. By the Einstein relation, $\sigma=q^{2} N\left(E_{F}\right) D(\tau)$ where $N\left(E_{F}\right)$ is the density of states at the Fermi level $E_{F}$, while $D(\tau)$ is the diffusion coefficient. One has $D(\tau)=L(\tau)^{2} / 3 \tau$ where $L(\tau)$ is the mean free path, namely the distance spread by a typical wave packet during the time $\tau$. Then (2) gives (3). Consequently, provided $\sigma_{\text {diff }}<1 / 2$ and $\tau$ depends on temperature as indicated above, the conductivity increases with temperature. For electrons in a periodic crystal, $\sigma_{\text {diff }}=1$ (ballistic quantum motion) and equation (3) gives a weak form of the Drude formula. 
Our aim in this paper is to put this type of argument on a rigorous ground. As we shall see, most of the work consists in defining the relevant mathematical framework liable to lead to (3). Then using many recent results on the characterization of singular continuous spectral measures, we will explain how to justify these arguments.

\subsection{Homogeneous media}

The first difficulty in dealing with materials such as quasicrystals is their lack of translation invariance at the atomic scale. In particular, there is no Bloch theorem allowing analytical calculations. However, these materials are homogeneous, namely they become translation invariant at a larger scale. In the limit where the sample size is large, it becomes simpler to represent these systems in the infinite volume limit. This is the first assumption made here. It means that we exclude the description of mesoscopic devices from our framework.

Our next assumption is to work within the one electron approximation, that is electronelectron and electron-phonon interactions are neglected in the Hamiltonian description and treated only in RTA by means of the collision time. This approximation is usually very good in metals because electrons can be described as quasiparticles dressed by interactions. As we have indicated previously, this is not a restriction when considering quasicrystals as long as the temperature is not smaller than $4 K$. Moreover, this framework includes electron-impurity scattering in the limit where imputities are quenched, namely their dynamic is not considered.

The third assumption concerns the energy range within which the electronic motion is studied. For indeed, only electrons with energies within $\mathcal{O}\left(k_{B} T\right)$ from the Fermi level contribute to the electronic transport. This allows to describe the electronic motion by means of an effective one-particle Hamiltonian, namely the restriction of the Schrödinger operator to this energy interval. In practice, it is possible to work within the so-called tight binding representation (see for instance [8]): we consider the nuclei as fixed on the vertices of the crystalline lattice $\mathcal{L}$ and we restrict ourselves to bands crossing the Fermi level; if only one band contributes to the Fermi level, wave functions are represented by a square summable sequence $\psi=(\psi(x))_{x \in \mathcal{L}}$, namely an element of $\mathcal{H}=\ell^{2}(\mathcal{L})$; the Hamiltonian is then a bounded selfadjoint operator $H=H^{*}$ on $\mathcal{H}$ whith off-diagonal matrix elements $\langle x|H| y\rangle$ decreasing exponentially fast to zero as $|x-y| \rightarrow \infty$; in practice, one considers only the nearest neighbors contribution to this operator. If $M$ bands must be taken into account, the wave function gets a band index $\psi=\left(\psi_{m}(x)\right), 1 \leq m \leq M$, and $\mathcal{H}=\ell^{2}(\mathcal{L}) \otimes \mathbf{C}^{M}$. In this way we avoid technicalities due to the unboundedness of the Schrödinger operator without restricting the physical domain of applicability. In order to avoid further irrelevant technical difficulties, we will considered in this paper systems in which $\mathcal{L}$ is a Bravais lattice of the type $\mathbf{Z}^{d}$. In the case of quasicrystals, this procedure must be slightly modified using groupoids (see for instance [8, 12, 45, 21]), but leads to a very similar description.

How can now homogeneity be described? Let us repeat the main arguments as given by one of the authors [8] leading to its mathematical definition: since the medium is translation invariant at large scale, translating $H$ should give the same physical properties. So there is no reason to prefer $H$ to any other of its translated. Moreover, changing from sample to sample is equivalent to looking at one unique infinite system through windows centered at different locations. This means that if we need to consider limits, $H$ is now by definition homogeneous if the strong closure of the set of all translated, denoted by $\Omega$, is compact. The choice of the strong topology is crucial: the norm topology leads to the too restricted notion of almost periodic operators while the weak topology would lead to a too wide notion of homogeneity. For 
an unbounded Hamiltonians, a similar construction holds when the strong resolvent topology is used [12]. $\Omega$ inherits a structure of a topological dynamical system by considering the action of the translation group on it. This dynamical system will be called the hull of $H$.

This framework will be used to describe robust, namely sample independent, physical properties. We will focus here on the measurement of space averages (or equivalently sample averages) of observables. By observable we mean any bounded operator obtained from the Hamiltonian through the elementary operations of translation, sums, product with a scalar, product, involution and limits in the strong topology. In this way the observable algebra contains no more than the energy and the homogeneity of the system. On the other hand, space averaging is not uniquely defined in general for its definition requires the choice of a translation invariant ergodic probability measure $\mathbf{P}$ on the hull. This choice will be arbitrary in what follows because all our results are independent of it. However, one could wonder whether there is a preferred choice in nature. This is probably related to the question of existence (and uniqueness) of a Gibbs measure with respect to the translation dynamic, but we will not address this question here.

These considerations lead to the construction of a $C^{*}$-algebra $\mathcal{A}$ that will be given in Sections 2.1 and 2.2 below. This $C^{*}$-algebra has been called the Non Commutative Brillouin Zone (NCBZ) of the homogeneous medium under consideration (see [8, 12]). In fact, in the periodic case, it coincides with the space of (matrix valued) continuous functions on the Brillouin zone. The probability $\mathbf{P}$ then defines a unique trace $\mathcal{T}$ on $\mathcal{A}$ which is nothing but the trace per unit volume. For a periodic crystal, it reduces to integration over the Brillouin zone.

One advantage of this formalism is that quantities computed through the trace $\mathcal{T}$ are insensitive to perturbations of the Hamiltonian by compact operators (see Theorem 11 below). This is actually a delicate point. A compact perturbation of the Hamiltonian can physically be interpreted as a localized impurity in the crystal. An experimentalist considers relevant only those properties that are insensitive to a given localized impurity, unless there is a definite procedure to faithfully reproduce this impurity from sample to sample. In homogeneous media, such a control is usually out of reach even for mesoscopic devices.

However, there are mathematical properties depending upon the occurence of a specific impurity. There have been quite a lot of works, starting with the result of Donoghue [26], showing that a rank one perturbation of a Hamiltonian with pure-point spectrum may produce a continuous spectrum (see e.g. [41] for a short review of historical references). One of the most striking results in this respect has been put in a systematic form in [71, 30, 60, 61. In particular, for the Anderson model in a regime of pure point spectrum with exponentially localized eigenstates, a topologically generic rank-one perturbation of the Hamiltonian produces a singular continuous spectrum [61]. Moreover, the authors of [61] show that the corresponding robust property is that the spectral and diffusion exponent do not change under such a perturbation and are equal to zero. This result is mathematically non trivial and remarkable. Their relevance in practical situation is however questionable.

In most experimental situations or numerical simulations, physicists are looking at sample independent quantities such as the average localization length, the conductance or the magnetoresistance. In more delicate questions, they may even look at universal fluctuations (a question not investigated here). In any case, the occurence of only a volume independent finite number of impurities is out of reach in practice. This is the main reason why we chose a mathematical framework in which delicate results, such as the topological instability of spectral properties by compact perturbations, can be discarded. 


\subsection{Spectral and transport exponents}

As we have seen in Section 1.1, scaling laws are the rule if anomalous transport occurs. This leads us to address the question of a proper definition of scaling exponents. Several inequivalent definitions are available in the literature dealing with fractal and multifractal analysis [65, 36, 35, 28, 52. In this work, we review several of them and discuss their relevance for our purpose. More precisely, we shall study the exponents characterizing the local behavior of the following three Borel measures: (i) the Density of States (DOS) expressing global properties related to the thermodynamics of the electron gas; (ii) the Local Density of States (LDOS), namely the spectral measure "with P-probability one"; (iii) the current-current correlation measure involved in Kubo's formula for the conductivity.

The basic definition of local exponents chosen here follows a suggestion of G. Mantica (see the second reference in [32]). Given a non-negative Lebesgue measurable function $f$ on $(0,1]$ we say $f(\epsilon) \underset{\epsilon \downarrow 0}{\sim} \epsilon^{\alpha}$ whenever $\int_{0}^{1} d \epsilon \epsilon^{-1-\gamma} f(\epsilon)$ converges for $\gamma<\alpha$ and diverges for $\gamma>\alpha$. If $f$ is monotonous, this is equivalent to $\alpha=\liminf _{\epsilon \rightarrow 0} \log f(\epsilon) / \log \epsilon$. A similar definition holds for the behavior at infinity. Note that this definition ignores all kinds of subdominant contributions and is likely to be robust. The local exponent $\alpha_{\nu}(E)$ of a Borel measure $\nu$ on $\mathbf{R}$ is introduced by

$$
\int_{E-\epsilon}^{E+\epsilon} d \nu\left(E^{\prime}\right) \underset{\epsilon \downarrow 0}{\sim} \epsilon^{\alpha_{\nu}(E)}
$$

We show that $E \mapsto \alpha_{\nu}(E)$ defines a function in $L^{\infty}(\mathbf{R}, d \nu)$ which depends only on the measure class of $\nu$ (see Theorem 2 below). Note that, although a consequence of standard measuretheoretic arguments, this result cannot be found in the literature [65, 81, 32, 28, 22, 40, 53, 20, 52, 48, 61, 61. Note further that other exponents defined in multifractal analysis do depend upon the measure in its own equivalence class (see Remark 11 in Section 3.3).

These properties are important in view of applications to homogeneous systems. A multifractal analysis of the DOS in the vicinity of the Fermi level may be useful for the thermodynamical properties of electron gas in our system because it gives more precise information about the DOS than the local exponents. However, multifractal properties of the LDOS are of no use in a homogeneous system where only the measure class of the spectral measure has some robustness. For indeed, by looking at the system through local windows chosen at random in the lattice, the corresponding spectral measures are in the same measure class and the entire equivalence class can be described in this way (with probability one). Therefore, we cannot expect exponents that do depend upon the spectral measure in its measure class to be relevant in practice.

The local exponents take values in the interval $[0,1] \nu$-almost surely. For an absolutely continuous measure $\nu, \alpha_{\nu}(E)=1 \nu$-almost surely. For a pure point measure $\nu, \alpha_{\nu}(E)=0 \nu$ almost surely. Hence these exponents allow to distinguish between different singular continuous measures. Examples of Hamiltonians with singular continuous spectra have been studied over years (see $4,469,7,31,24,19,75,76,9,10,25,11,18,39,44)$ and the question of computing their spectral exponents is certainly worth of study [43. The local exponents can be computed both numerically and analytically by using the Green's function [82] (see also [61]):

$$
\Im m \int_{\mathbf{R}} \frac{d \nu\left(E^{\prime}\right)}{E^{\prime}-(E-\imath \epsilon)} \underset{\epsilon \downarrow 0}{\sim} \epsilon^{\alpha_{\nu}(E)-1} .
$$


Note, however, that numerical computations may concern exceptional values of $E$ for which $\alpha_{\nu}(E)$ is larger than 1 .

The definition of local exponents extend to the spectral analysis of a self-adjoint operator on a separable Hilbert space. We show that the exponents are invariants of the operator itself independent of the states in the Hilbert space. For covariant families of such operators arising in homogeneous media as discussed in Section 1.2, the exponents are moreover $\mathbf{P}$-almost surely constant and define the exponents $\alpha_{\text {LDOS }}(E)$ of the LDOS. The DOS is always regular with respect to the LDOS in the sense that for typical energies one has $\alpha_{\mathrm{LDOS}}(E) \leq \alpha_{\mathrm{DOS}}(E)$.

The definition of the diffusion exponent $\sigma_{\text {diff }}$ given in equation (2) is generalized by restricting the dynamics to an energy interval $\Delta$ and by extending it to the case of homogeneous systems. One talks of ballistic motion whenever $\sigma_{\text {diff }}(\Delta)=1$ and of regular diffusion whenever $\sigma_{\text {diff }}(\Delta)=$ 1/2. Localization, a behavior strictly stronger than $\sigma_{\text {diff }}(\Delta)=0$, has been studied in [13, 14] and will be discussed in more detail in Section 2.6. For any other value of $\sigma_{\text {diff }}$, the quantum diffusion is called anomalous. Guarneri's inequality [32] (see also [20, 33, 48, 6]) gives a lower bound of the diffusion exponent by the local exponents of the LDOS:

$$
\alpha_{\mathrm{LDOS}} \leq d \cdot \sigma_{\mathrm{diff}}
$$

where $d$ is the space dimension (see Chapter 2 for a precise formulation).

Guarneri's inequality has several direct physical implications [15]. For dimension one, an absolutely continuous spectrum implies ballistic quantum motion. However, for $d \geq 2$, one may have both absolutely continuous spectrum and quantum diffusion with $\sigma_{\text {diff }} \geq 1 / d$. This is expected, in particular, for the three-dimensional Anderson model at low disorder where, on the basis of the renormalization group calculation, the diffusion exponent is conjectured to be $\sigma_{\text {diff }}=1 / 2$ [77, 1]. The same situation is expected for the Anderson model in dimension two provided spin-orbit coupling is added [37]. For three-dimensional quasicrystals, Guarneri's inequality allows a diffusion exponent as low as $1 / 3$ without forbidding an absolutely continuous spectrum. In Chapter 5, we give an example of a model having $\sigma_{\text {diff }}=1 / 2$. Note that this model is an operator theoretic version of the so-called coherent potential approximation of the Anderson model [51].

\subsection{Overview of this article}

After this motivating introduction, we have organized this article as follows. Chapter 2 contains the main results and discriminates between the new results and the ones already obtained elsewhere. In Sections 2.1 and 2.2 we give an account of the mathematical framework introduced in [8, 12 and used to describe homogeneous media. We also give a precise formulation to the stability under compact perturbations of the Hamiltonian. Both well-known and new results on local spectral exponents of Borel measures are presented in Section 2.3 before being extended to self-adjoint operators in Section 2.4. In Sections 2.5 through 2.8 are devoted to the exponents of the LDOS, DOS and current-current correlation function as well as the anomalous Drude formula.

Chapter 3 contains proofs of the results of Sections 2.3 and 2.4 as well as some complementary results. In particular, in Sections 3.3 and 3.4 we give few known or less known facts about multifractal analysis which are of interest.

The remaining results of Chapter 2, all linked to homogeneous systems, are proved in Chapter 1 . The content of this chapter has not yet been treated in the literature. 
Chapter 5 is devoted to the calculation of the diffusion exponent in the Anderson model with free random variables [51].

An Appendix completes the study of the hull whenever an impurity (or a compact perturbation) is added to the Hamiltonian.

\section{Notations and results}

\subsection{Construction and stability of the hull}

Let $\hat{H}=\hat{H}^{*}$ be a bounded Hamiltonian acting on the one-particle Hilbert space $\mathcal{H}=\ell^{2}\left(\mathbf{Z}^{d}\right)$. Let us consider its hull $\Omega_{\hat{H}}$ given by

$$
\Omega_{\hat{H}}={\overline{\left\{U(a) \hat{H} U(a)^{-1} \mid a \in \mathbf{Z}^{d}\right\}}}^{s},
$$

where $(U(a))_{a \in \mathbf{Z}^{d}}$ is a projective unitary representation of $\mathbf{Z}^{d}$ on $\mathcal{H}$ and the closure is taken with respect to the strong operator topology. By definition $\hat{H}$ is homogeneous if $\Omega_{\hat{H}}$ is a compact metrisable space [8, 12]. The projective representation $U$ induces a $\mathbf{Z}^{d}$-action $T$ on $\Omega_{\hat{H}}$ by homeomorphisms. Each point $\omega \in \Omega_{\hat{H}}$ describes a disorder or aperiodicity configuration of the crystal. A $T$-invariant and ergodic probability measure $\mathbf{P}$ on $\Omega_{\hat{H}}$ gives the probability with which specific configurations are realized. We now have the following stability theorem showing that any quantity defined almost surely with respect to $\mathbf{P}$ is stable with respect to compact perturbations of the Hamiltonian $\hat{H}$.

Theorem 1 Let $\hat{V}=\hat{V}^{*}$ be a compact operator. If $\hat{H}$ is homogeneous, so is $\hat{H}+\hat{V}$. Then the symmetric difference of the compact hulls $\Omega_{\hat{H}} \triangle \Omega_{\hat{H}+\hat{V}}$ is at most countable. Moreover, any $T$ invariant measure on $\Omega_{\hat{H}}$ or $\Omega_{\hat{H}+\hat{V}}$ has its support in $\Omega_{\hat{H}} \cap \Omega_{\hat{H}+\hat{V}}$. Hence an invariant measure on $\Omega_{\hat{H}}$ completely determines an invariant measure on $\Omega_{\hat{H}+\hat{V}}$.

The proof is given in the appendix. In the sequel, we will drop the index $\hat{H}$ in $\Omega_{\hat{H}}$ whenever there is no ambiguity.

\subsection{The non-commutative Brillouin zone}

In the last section, we constructed the hull of the Hamiltonian $\hat{H}$. Let us briefly review the construction of the corresponding crossed product $C^{*}$-algebra $\mathcal{A}$ called the non-commutative Brillouin zone (NCBZ) of $\hat{H}[8,[12$. For quasicrystals, the algebra is in general given by a $C^{*}$-algebra associated to a groupoid [12, 45, 21]. In that case the formulæ below have direct analogs except for Birkhoff's theorem (equation (8)) which is not yet proved in that context as far as we know. All the analysis of this article should transpose directly to that case.

Let us first consider the topological vector space $\mathcal{C}_{\kappa}\left(\Omega \times \mathbf{Z}^{d}\right)$ of continuous functions with compact support on $\Omega \times \mathbf{Z}^{d}$. It is endowed with the following structure of a ${ }^{*}$-algebra by

$$
A B(\omega, n)=\sum_{l \in \mathbf{Z}^{d}} A(\omega, l) B\left(T^{-l} \omega, n-l\right) e^{\frac{\imath q}{2 \hbar} \mathcal{B} . n \wedge l}, \quad A^{*}(\omega, n)=\overline{A\left(T^{-n} \omega,-n\right)}
$$


where $A, B \in \mathcal{C}_{\kappa}\left(\Omega \times \mathbf{Z}^{d}\right), \omega \in \Omega, n \in \mathbf{Z}^{d}$, finally the antisymmetric real tensor $\mathcal{B}=\left(B_{i, j}\right)$ is a uniform magnetic field and $\mathcal{B} . n \wedge l=\sum_{i, j} B_{i, j} n_{i} l_{j}$. For $\omega \in \Omega$, this ${ }^{*}$-algebra is represented on $\mathcal{H}=\ell^{2}\left(\mathbf{Z}^{d}\right)$ by

$$
\pi_{\omega}(A) \psi(n)=\sum_{l \in \mathbf{Z}^{d}} A\left(T^{-n} \omega, l-n\right) e^{\frac{\imath q}{2 \hbar} \mathcal{B} . l \wedge n} \psi(l), \quad \psi \in \ell^{2}\left(\mathbf{Z}^{d}\right),
$$

namely, $\pi_{\omega}$ is linear, $\pi_{\omega}(A B)=\pi_{\omega}(A) \pi_{\omega}(B)$ and $\pi_{\omega}(A)^{*}=\pi_{\omega}\left(A^{*}\right)$. In addition, $\pi_{\omega}(A)$ is a bounded operator. Let a projective unitary representations $(U(a))_{a \in \mathbf{Z}^{d}}$ on $\mathcal{H}$ be given by the magnetic translations:

$$
U(a) \psi(n)=e^{\frac{2 q}{2 \hbar} \mathcal{B} \cdot a \wedge n} \psi(n-a) .
$$

Then the representations are related by the covariance condition

$$
U(a) \pi_{\omega}(A) U(a)^{-1}=\pi_{T^{a} \omega}(A), \quad a \in \mathbf{Z}^{d} .
$$

Now $\|A\|=\sup _{\omega \in \Omega}\left\|\pi_{\omega}(A)\right\|$ defines a $C^{*}$-norm. This allows to define $\mathcal{A}=C^{*}\left(\Omega \times \mathbf{Z}^{d}, \mathcal{B}\right)$ as the completion of $\mathcal{C}_{\kappa}\left(\Omega \times \mathbf{Z}^{d}\right)$ under this norm. Clearly, the representations $\pi_{\omega}$ can be continuously extended to this $C^{*}$-algebra. This family of representations is strongly continuous in $\omega$ for any fixed $A \in \mathcal{A}$. Finally, there exists an element $H \in \mathcal{A}$ such that $\pi_{\omega_{0}}(H)=\hat{H}$ where $\omega_{0}$ is the point $\hat{H}$ of $\Omega$ [12].

Given an invariant and ergodic probability measure $\mathbf{P}$ on $\Omega$, a trace $\mathcal{T}$ on all $\mathcal{A}$ is defined by

$$
\mathcal{T}(A)=\int_{\Omega} d \mathbf{P}(\omega)\left\langle 0\left|\pi_{\omega}(A)\right| 0\right\rangle=\lim _{l \rightarrow \infty} \frac{1}{\left|\Lambda_{l}\right|} \sum_{n \in \Lambda_{l}}\left\langle n\left|\pi_{\omega^{\prime}}(A)\right| n\right\rangle,
$$

where $|n\rangle$ is the state completely localized at $n \in \mathbf{Z}^{d}$. The $\Lambda_{l}$ 's are an increasing sequence of rectangles centered at the origin. The equality holds for almost all $\omega^{\prime}$ by Birkhoff's ergodic theorem. This shows that $\mathcal{T}$ is the trace per unit volume. Note that a compact perturbation of the Hamiltonian changes the $C^{*}$-algebra $\mathcal{A}$, but not the trace per unit volume of observables.

$\mathcal{T}$ gives rise to the GNS Hilbert space $L^{2}(\mathcal{A}, \mathcal{T})$ and GNS representation $\pi_{\mathrm{GNS}}$. We denote by $L^{\infty}(\mathcal{A}, \mathcal{T})$ the von Neumann algebra $\pi_{\mathrm{GNS}}(\mathcal{A})^{\prime \prime}$ where " is the bicommutant. By a theorem of Connes [14, $L^{\infty}(\mathcal{A}, \mathcal{T})$ is canonically isomorphic to the von Neumann algebra of $\mathbf{P}$-essentially bounded, weakly measurable and covariant families $A_{\omega}$ of operators on $\mathcal{H}=\ell^{2}\left(\mathbf{Z}^{2}\right)$ endowed with the norm

$$
\|A\|_{L^{\infty}}=\mathbf{P}-\underset{\omega \in \Omega}{\operatorname{essinf}}\left\|A_{\omega}\right\|_{\mathcal{B}(\mathcal{H})}
$$

Consequently, the family of representations $\pi_{\omega}$ extends to a family of weakly measurable representations of $L^{\infty}(\mathcal{A}, \mathcal{T})$. Moreover, the trace $\mathcal{T}$ extends to $L^{\infty}(\mathcal{A}, \mathcal{T})$.

To define a differential structure on $\mathcal{A}$, consider the family of $*$-automorphisms $\rho_{k_{j}}$ of $\mathcal{A}$ given by

$$
\left(\rho_{k_{j}} A\right)(\omega, n)=e^{\imath k_{j} n_{j}} A(\omega, n), \quad A \in \mathcal{A} .
$$

Then the $d$ generators of $\rho_{k_{j}}$, denoted by $\partial_{j}, j=1 \ldots d$, are $*$-derivations. We use the notation $\vec{\nabla}=\left(\partial_{1} \ldots \partial_{d}\right)$. If $\vec{X}=\left(X_{1}, \ldots, X_{d}\right)$ is the position operator in $\mathcal{H}=\ell^{2}\left(\mathbf{Z}^{d}\right)$,

$$
\left(X_{j} \phi\right)(n)=n_{j} \phi(n), \quad \phi \in \ell^{2}\left(\mathbf{Z}^{d}\right), n=\left(n_{1}, \ldots, n_{d}\right) \in \mathbf{Z}^{d}
$$


one can check that

$$
\pi_{\omega}\left(\rho_{k_{j}}(A)\right)=e^{\imath k_{j} X_{j}}\left(\pi_{\omega}(A)\right) e^{-\imath k_{j} X_{j}},
$$

and $\pi_{\omega}(\vec{\nabla} A)=\imath\left[\vec{X}, \pi_{\omega}(A)\right]$. The differential elements of $\mathcal{A}$ are

$$
\mathcal{C}^{k}(\mathcal{A})=\left\{A \in \mathcal{A} \mid \partial_{j_{1}} \cdots \partial_{j_{l}} A \in \mathcal{A}, j_{1}, \ldots, j_{l} \in 1, \ldots, d, l \leq k\right\}
$$

\subsection{Local exponents of Borel measures}

Definition 1 Let $f$ and $g$ be Lebesgue-measurable non-negative functions on the intervals $(0, b]$ and $[b, \infty)$ respectively, $b>0$. The behaviors $f(x) \underset{x \downarrow 0}{\sim} x^{\alpha}$ and $g(x) \underset{x \uparrow \infty}{\sim} x^{\eta}$ are defined by

$$
\begin{gathered}
\alpha=\sup \left\{\gamma \in \mathbf{R} \mid \exists a \in \mathbf{R}: 0<a \leq b ; \int_{0}^{a} \frac{d x}{x} \frac{f(x)}{x^{\gamma}}<\infty\right\}, \\
\eta=\inf \left\{\gamma \in \mathbf{R} \mid \exists a \in \mathbf{R}: b \leq a<\infty ; \int_{a}^{\infty} \frac{d x}{x} \frac{g(x)}{x^{\gamma}}<\infty\right\}
\end{gathered}
$$

Let $\mathcal{M}$ be the space of Borel probability measures on $\mathbf{R}$ with the vague topology.

Definition 2 Let $\nu$ be in $\mathcal{M}$ and $E \in \mathbf{R}$. The local exponent $\alpha_{\nu}(E)$ of $\nu$ at $E$ is defined by

$$
\int_{E-\epsilon}^{E+\epsilon} d \nu\left(E^{\prime}\right) \underset{\epsilon \downarrow 0}{\sim} \epsilon^{\alpha_{\nu}(E)}
$$

Remark that the local exponents are always bigger than or equal to 0 . Note further that Fubini's theorem immediately implies that

$$
\alpha_{\nu}(E)=\sup \left\{\gamma \in \mathbf{R} \mid \int d \nu\left(E^{\prime}\right) \frac{1}{\left|E-E^{\prime}\right|^{\gamma}}<\infty\right\}
$$

Theorem 2 Let $\nu, \mu \in \mathcal{M}$.

i) For $\nu$-almost all $E, 0 \leq \alpha_{\nu}(E) \leq 1$.

ii) If $\mu$ dominates $\nu$, then $\alpha_{\mu}(E) \leq \alpha_{\nu}(E) \mu$-almost surely and $\alpha_{\nu}(E)=\alpha_{\mu}(E) \nu$-almost surely.

iii) If $\nu$ is pure-point, then $\nu$-almost surely $\alpha_{\nu}(E)=0$.

iv) If $\nu$ is absolutely continuous, then $\alpha_{\nu}(E)=1 \nu$-almost surely.

v) $(\nu, E) \in \mathcal{M} \times \mathbf{R} \mapsto \alpha_{\nu}(E)$ is a Borel function.

This result shows that $\nu$-almost surely in $E$, the local exponent $\alpha_{\nu}(E)$ only depends upon the measure class of $\nu$. Note that item ii) does not follow directly from the Radon-Nykodim theorem. For example, let $f(E) d E, \in L^{1}(\mathbf{R})$, be an absolutely continuous measure. The function $f$ may have singularities where the exponent is smaller than 1 . However, according to Theorem 2, these points only have Lebesgue measure 0. Note also that $\alpha_{\nu}(E)$ may be bigger than 1 , but only on a set of zero $\nu$-measure. In practice, one can use the following characterization which is an extension of the Charles de la Vallée Poussin theorem [57]. The proof can be found in [82], see also [61]. 
Theorem 3 Let $\nu \in \mathcal{M}$ and $G_{\nu}(z)=\int_{\mathbf{R}} \frac{d \nu\left(E^{\prime}\right)}{z-E^{\prime}}$, $\Im m(z)>0$, its Green's function. The exponent $\beta(E)$ is introduced by

$$
\Im m\left(G_{\nu}(E-\imath \epsilon)\right) \underset{\epsilon \downarrow 0}{\sim} \epsilon^{\beta(E)}
$$

Then $\beta(E)=\alpha_{\nu}(E)-1$ whenever $\alpha_{\nu}(E) \in[0,2]$.

The previous results allow to associate to a given measure class $[\nu]$ a function $\alpha_{\nu} \in$ $L^{\infty}(\mathbf{R}, d \nu)$ taking values in the interval $[0,1] \nu$-almost surely. Of particular interest are the biggest and smallest typical exponent in a given Borel set (see [81]).

Definition 3 Let $\nu \in \mathcal{M}$ and $\Delta \subset \mathbf{R}$ be a Borel set. The upper and lower essential exponents are defined by

$$
\alpha_{\nu}^{+}(\Delta)=\nu-\operatorname{esssup}_{E \in \Delta} \alpha_{\nu}(E), \quad \alpha_{\nu}^{-}(\Delta)=\nu-\underset{E \in \Delta}{\operatorname{essinf}} \alpha_{\nu}(E)
$$

Corollary 1 Let $\mu, \nu$ be two Borel measures on $\mathbf{R}$ and $\Delta \subset \mathbf{R}$ a Borel set. If $\mu$ dominates $\nu$, then $\alpha_{\nu}^{+}(\Delta) \leq \alpha_{\mu}^{+}(\Delta)$ and $\alpha_{\mu}^{-}(\Delta) \leq \alpha_{\nu}^{-}(\Delta)$. If $\mu$ and $\nu$ are in the same measure class, then $\alpha_{\mu}^{+}(\Delta)=\alpha_{\nu}^{+}(\Delta)$ and $\alpha_{\mu}^{-}(\Delta)=\alpha_{\nu}^{-}(\Delta)$.

Proposition 1 Let $\Delta \subset \mathbf{R}$ be a Borel set, then $\nu \in \mathcal{M} \mapsto \alpha_{\nu}^{+}(\Delta)$ and $\nu \in \mathcal{M} \mapsto \alpha_{\nu}^{-}(\Delta)$ are Borel functions.

The essential exponents are linked to the Hausdorff dimensions $\operatorname{dim}_{\mathrm{H}}$ (see for example 28]) associated to the measure $\nu$ and its support by the following theorem of Rodgers and Taylor [65] (our formulation only slightly varies from theirs).

Theorem [65] Let $\nu \in \mathcal{M}$ and $\Delta \subset \mathbf{R}$ Borel. If $S_{0}$ is the Borel set defined by

$$
S_{0}=\left\{E \in \Delta \mid \alpha_{\nu}(E) \leq \alpha_{\nu}^{+}(\Delta)\right\},
$$

then $\nu\left(S_{0}\right)=\nu(\Delta)$ and $\operatorname{dim}_{\mathrm{H}}\left(S_{0}\right)=\alpha_{\nu}^{+}(\Delta)$. There is no Borel set $S \subset \Delta$ with $\operatorname{dim}_{\mathrm{H}}(S)<\alpha_{\nu}^{+}(\Delta)$ satisfying $\nu(S)=\nu(\Delta)$. Moreover, if a Borel set $S \subset \Delta$ satisfies $\operatorname{dim}_{\mathrm{H}}(S)<\alpha_{\nu}^{-}(\Delta)$, then $\nu(S)=0$.

The Hausdorff dimension of a measure is defined by [81]

$$
\operatorname{dim}_{\mathrm{H}}\left(\left.\nu\right|_{\Delta}\right)=\inf _{S \subset \Delta}\left\{\operatorname{dim}_{\mathrm{H}}(S) \mid \nu(S)=\nu(\Delta)\right\}
$$

where the infimum is taken over Borel sets $S \subset \Delta$. Rodgers' and Taylors theorem shows that $\operatorname{dim}_{\mathrm{H}}\left(\left.\nu\right|_{\Delta}\right)=\alpha_{\nu}^{+}(\Delta)$. 


\subsection{Local exponents of a self-adjoint operator}

Let $H$ be a selfadjoint operator acting on the separable Hilbert space $\mathcal{H}$. The spectral theory associates to $H$ a $\mathcal{H}$-projection-valued Borel measure $\Pi$ on $\mathbf{R}$ [57]. Furthermore, for any $\phi \in \mathcal{H}$, $\|\phi\|=1$, let $\rho_{\phi}$ be the spectral measure of $H$ relative to $\phi$, namely for $f \in C_{0}(\mathbf{R})$,

$$
\int d \rho_{\phi}(E) f(E)=\langle\phi|f(H)| \phi\rangle=\int\langle\phi|\Pi(d E)| \phi\rangle f(E) .
$$

In physics literature, $\rho_{\phi}$ is called the local density of states (LDOS).

Definition 4 Let $E \in \mathbf{R}$ and $\Delta$ be a Borel subset of $\mathbf{R}$. The spectral exponent and essential spectral exponents of $\Pi($ or $H)$ are defined by

$$
\alpha_{\Pi}(E)=\inf _{\phi \in \mathcal{H}} \alpha_{\rho_{\phi}}(E), \quad \alpha_{\Pi}^{+}(\Delta)=\sup _{\phi \in \mathcal{H}} \alpha_{\rho_{\phi}}^{+}(\Delta), \quad \alpha_{\Pi}^{-}(\Delta)=\inf _{\phi \in \mathcal{H}} \alpha_{\rho_{\phi}}^{-}(\Delta)
$$

Theorem 4 There exists $\psi \in \mathcal{H}$ with

$$
\alpha_{\Pi}^{+}(\Delta)=\alpha_{\rho_{\psi}}^{+}(\Delta), \quad \alpha_{\Pi}^{-}(\Delta)=\alpha_{\rho_{\psi}}^{-}(\Delta) .
$$

This result shows that there are typical states in $\mathcal{H}$ giving the generic properties of the spectrum.

\subsection{Density of states and local density of states}

Let $H \in \mathcal{A}$ be the Hamiltonian. The spectral projection of $\pi_{\omega}(H)$ is denoted by $\Pi_{\omega}$.

Theorem 5 For $E \in \mathbf{R}$ and a Borel subset $\Delta \subset \mathbf{R}$, the exponents $\alpha_{\Pi_{\omega}}(E), \alpha_{\Pi_{\omega}}^{+}(\Delta)$ and $\alpha_{\Pi_{\omega}}^{-}(\Delta)$ are $\mathbf{P}$-almost surely independent of $\omega$. The common values are denoted by $\alpha_{\mathrm{LDOS}}(E)$, $\alpha_{\mathrm{LDOS}}^{+}(\Delta)$ and $\alpha_{\mathrm{LDOS}}^{-}(\Delta)$ respectively.

A related result was proved by Last 48]. Theorem 1 implies that all these exponents are stable with respect to compact perturbation of the Hamiltonian.

Corollary 2 If $\pi_{\omega}(H)$ has pure-point spectrum in $\Delta, \mathbf{P}$-almost surely, then $\alpha_{\mathrm{LDOS}}^{ \pm}(\Delta)=0$. If $\pi_{\omega}(H)$ has absolutely continuous spectrum in $\Delta$ for $\mathbf{P}$-almost all $\omega \in \Omega$, then $\alpha_{\mathrm{LDOS}}^{ \pm}(\Delta)=1$. If $0<\alpha_{\mathrm{LDOS}}^{-}(\Delta) \leq \alpha_{\mathrm{LDOS}}^{+}(\Delta)<1$, then the spectrum is singular continuous in $\Delta$ for $\mathbf{P}$-almost all $\omega \in \Omega$.

Another important spectral measure associated to $H$ is the density of states (DOS) defined by

$$
\int d \mathcal{N}(E) f(E)=\int d \mathbf{P}(\omega)\left\langle 0\left|\pi_{\omega}(f(H))\right| 0\right\rangle, \quad f \in C_{0}(\mathbf{R}) .
$$

We denote $\alpha_{\text {Dos }}(E)=\alpha_{\mathcal{N}}(E)$ and for any Borel subset $\Delta$ of $\mathbf{R}$, we set:

$$
\alpha_{\mathrm{DOS}}^{ \pm}(\Delta)=\alpha_{\mathcal{N}}^{ \pm}(\Delta)
$$

Theorem 6 For $E \in \mathbf{R}$ and a Borel subset $\Delta \subset \mathbf{R}$,

$$
\alpha_{\mathrm{LDOS}}(E) \leq \alpha_{\mathrm{DOS}}(E), \quad \alpha_{\mathrm{LDOS}}^{ \pm}(\Delta) \leq \alpha_{\mathrm{DOS}}^{ \pm}(\Delta),
$$

Note that this implies the Hausdorff dimension of the DOS is bigger than or equal to the Hausdorff dimension of the LDOS. 


\subsection{Diffusion exponents and localization}

This section is devoted to dynamical quantum diffusion and quantum localization. The diffusion exponent allows to measure the importance of quantum interference effects due to the frozen (disorder or quasiperiodic) potential in the one-particle Hamiltonian. Collisions with timedependent disorder such as collisions with phonons and its effects on diffusion are not considered here; in RTA these effects are treated by the phenomenological constant $\tau_{\text {rel }}$ in Kubo's formula. Diffusion is supposed to be isotropic here, but this is only done for sake of notational simplicity.

For a given Borel set $\Delta \subset \mathbf{R}$, the mean square displacement operator is

$$
\delta X_{\omega, \Delta}^{2}(T)=\int_{0}^{T} \frac{d t}{T} \Pi_{\omega}(\Delta)\left(\vec{X}_{\omega}(t)-\vec{X}\right)^{2} \Pi_{\omega}(\Delta),
$$

where $\vec{X}_{\omega}(t)=e^{\imath t \pi_{\omega}(H)} \vec{X} e^{-\imath t \pi_{\omega}(H)}$.

Definition 5 The diffusion exponent $\sigma_{\text {diff }}(\Delta)$ is defined by

$$
\int_{\Omega} d \mathbf{P}(\omega)\left\langle 0\left|\delta X_{\omega, \Delta}^{2}(T)\right| 0\right\rangle \underset{T \uparrow \infty}{\sim} T^{2 \sigma_{\mathrm{diff}}(\Delta)} .
$$

Proposition 2 Let $\Pi(\Delta)=\chi_{\Delta}(H) \in L^{\infty}(\mathcal{A}, \mathcal{T})$ where $\chi_{\Delta}$ is the characteristic function on the Borel set $\Delta$ and suppose that $H \in \mathcal{C}^{1}(\mathcal{A})$. Then

$$
\int_{0}^{T} \frac{d t}{T} \mathcal{T}\left(\left|\vec{\nabla}\left(e^{-\imath H t}\right)\right|^{2} \Pi(\Delta)\right)=\int_{\Omega} d \mathbf{P}(\omega)\left\langle 0\left|\delta X_{\omega, \Delta}^{2}(T)\right| 0\right\rangle \underset{T \uparrow \infty}{\sim} T^{2 \sigma_{\text {diff }}(\Delta)} .
$$

Theorem 7 Let $H \in \mathcal{C}^{1}(\mathcal{A})$. Then:

i) $0 \leq \sigma_{\text {diff }}(\Delta) \leq 1$.

ii) Let $\hat{V}$ be a compact operator on $\mathcal{H}=\ell^{2}\left(\mathbf{Z}^{d}\right)$ such that $[\vec{X}, \hat{V}]$ is bounded. Let the invariant ergodic measure on $\Omega_{\hat{H}}$ determine that on $\Omega_{\hat{H}+\hat{V}}$ as in Theorem $\mathbb{Q}$, then the diffusion exponents $\sigma_{\text {diff }}(\Delta)$ of $\hat{H}$ and $\hat{H}+\hat{V}$ are equal.

iii) Guarneri's bound: for any open interval $\Delta \subset \mathbf{R}$ :

$$
\alpha_{\mathrm{LDOS}}^{+}(\Delta) \leq d \cdot \sigma_{\mathrm{diff}}(\Delta),
$$

whenever $H \in \mathcal{C}^{k}(\mathcal{A})$ for some $k>d / 2$.

An inequality between spectral and diffusion exponents was first proved by Guarneri [32]. A further contribution is due to Combes [20]. Last improved the proof in order to show that it is the most continuous part of the spectrum which gives the lower bound of the diffusion exponent [48], see also [6]. The bound (16) links exponents associated to the covariant family of Hamiltonians irrespective of the choice of a specific vector in Hilbert space.

Let us conclude this section with a discussion of localization. The following localization criterion for a Borel subset $\Delta \subset \mathbf{R}$ was introduced in [13] motivated by the study of the quantum Hall effect [14]:

$$
l^{2}(\Delta)=\limsup _{T \rightarrow \infty} \int_{0}^{T} \frac{d t}{T} \mathcal{T}\left(\left|\vec{\nabla} e^{-\imath H t}\right|^{2} \Pi(\Delta)\right)<\infty .
$$


Note that it is strictly stronger than $\sigma_{\text {diff }}(\Delta)=0$ because no logarithmic divergencies are allowed. Actually, (17) coincides with the localization criterion used by physicists: in physics literature, averages of products of Green functions are used; this leads to the current-current correlation measure $m$ below (Theorem 11). In the Anderson model and a wide class of other models, the condition (17) has been shown to hold for the spectral subsets generally considered to be localized [14].

Theorem 8 Suppose that the localization condition (17) is satisfied for a Borel set $\Delta \subset \mathbf{R}$. Then the following holds:

i) [13, 14] $\sigma_{\text {diff }}(\Delta)=0$ and $\pi_{\omega}(H)$ has pure-point spectrum in $\Delta$ for $\mathbf{P}$-almost every $\omega \in \Omega$.

ii) Let $\hat{V}$ be a compact self-adjoint operator such that $[\vec{X}, \hat{V}]$ is bounded. Let the invariant ergodic measure on $\Omega_{\hat{H}}$ determine that on $\Omega_{\hat{H}+\hat{V}}$ as in Theorem $\mathbb{1}$, then the localization condition (17) is simultaneously satisfied for $\hat{H}$ and $\hat{H}+\hat{V}$.

iii) 13, 14] There is a $\mathcal{N}$-measurable function l on $\Delta$ such that for every Borel subset $\Delta^{\prime}$ of $\Delta$ :

$$
l^{2}\left(\Delta^{\prime}\right)=\int_{\Delta^{\prime}} d \mathcal{N}(E) l(E)^{2}
$$

Let us notice that the criterion (17) can be weakened in the following way: let $g$ be any increasing function on $\mathbf{R}^{+}$such that $\lim _{x \rightarrow \infty} g(x)=\infty$ and consider

$$
l_{g}(\Delta)=\limsup _{T \rightarrow \infty} \int_{0}^{T} \frac{d t}{T} \int_{\Omega} d \mathbf{P}(\omega)\left\langle 0\left|\Pi_{\omega}(\Delta) g\left(\left|\vec{X}_{\omega}(t)-\vec{X}\right|\right) \Pi_{\omega}(\Delta)\right| 0\right\rangle .
$$

Then $l_{g}(\Delta)<\infty$ suffices to get pure-point spectrum in $\Delta, \mathbf{P}$-almost surely and to insure that this property is stable by compact perturbations of the Hamiltonian.

\subsection{Current-current correlation function}

In this section we give some useful formulæ for the calculation of the diffusion exponent. As illustrative application, the diffusion exponent of Wegner's $n$-orbital model is calculated in Chapter 5. The current operator is defined (if $H \in \mathcal{C}^{1}(\mathcal{A})$ ) by

$$
\vec{J}=\vec{\nabla}(H) .
$$

The current-current correlation functions are the Borel measures $m_{i, j}$ on $\mathbf{R}^{2}$ given by 477

$$
\int_{\mathbf{R}^{2}} d m_{i, j}\left(E, E^{\prime}\right) f(E) g\left(E^{\prime}\right)=\mathcal{T}\left(\partial_{i}(H) f(H) \partial_{j}(H) g(H)\right),
$$

where $f, g \in C_{0}(\mathbf{R})$. The right hand side defines a positive and continuous bilinear form on $C_{0}(\mathbf{R}) \times C_{0}(\mathbf{R}) \times M_{d}(\mathbf{C})$. The Riesz-Markov theorem [57 then assures the existence of the Radon measures $m_{i, j}$ on $\mathbf{R}^{2}$ with finite mass. The cyclicity of the trace induces the following symmetry of $m_{j, j}$ with respect to the diagonal $E=E^{\prime}$ :

$$
\int_{\mathbf{R}^{2}} d m_{j, j}\left(E, E^{\prime}\right) f\left(E, E^{\prime}\right)=\int_{E \geq E^{\prime}} d m_{j, j}\left(E, E^{\prime}\right)\left(f\left(E, E^{\prime}\right)+f\left(E^{\prime}, E\right)\right) .
$$

The isotropic part $m$ is the measure $m=\sum_{j=1}^{d} m_{j, j} / d$. It is called the current-current correlation measure or also the conductivity measure. It allows to calculate the diffusion exponent. 
Theorem 9 Given a Borel set $\Delta \subset \mathbf{R}$ and $\epsilon>0$, let $\operatorname{diag}(\Delta, \epsilon)$ be the set of points in $\Delta \times \mathbf{R}$ within distance $\epsilon$ from the diagonal in $\mathbf{R}^{2}$, then

$$
\int_{\operatorname{diag}(\Delta, \epsilon)} d m\left(E, E^{\prime}\right) \underset{\epsilon \downarrow 0}{\sim} \epsilon^{2\left(1-\sigma_{\text {diff }}(\Delta)\right)}
$$

The Stieltjes transform of $m$ is given by

$$
S_{m}\left(z_{1}, z_{2}\right)=\frac{1}{(2 \pi \imath)^{2}} \int_{\mathbf{R}^{2}} d m\left(E, E^{\prime}\right) \frac{1}{\left(E-z_{1}\right)\left(E^{\prime}-z_{2}\right)} .
$$

If $H=H_{0}+V$ with a translation invariant kinetic part $H_{0}$ and a potential $V$ satisfying $\vec{\nabla}(V)=0$, then $S_{m}$ can be calculated by means of the 2-point Green's function:

$$
S_{m}\left(z_{1}, z_{2}\right)=\frac{1}{d} \frac{1}{(2 \pi \imath)^{2}} \sum_{r, s, t \in \mathbf{Z}^{d}}\left\langle 0\left|\vec{\nabla}\left(H_{0}\right)\right| r\right\rangle \cdot\left\langle s\left|\vec{\nabla}\left(H_{0}\right)\right| t\right\rangle G^{2}\left(z_{1}, z_{2}, r, s, t, 0\right)
$$

where

$$
G^{2}\left(z_{1}, z_{2}, r, s, s^{\prime}, r^{\prime}\right)=\int_{\Omega} d \mathbf{P}(\omega)\left\langle r\left|\frac{1}{z_{1}-\pi_{\omega}(H)}\right| s\right\rangle\left\langle s^{\prime}\left|\frac{1}{z_{2}-\pi_{\omega}(H)}\right| r^{\prime}\right\rangle
$$

Theorem 10 The diffusion exponent is given by

$$
\Re e\left(\int_{\mathbf{R}} d a S_{m}(a+\imath \epsilon, a-\imath \epsilon)\right) \underset{\epsilon \downarrow 0}{\sim} \epsilon^{1-2 \sigma_{\mathrm{diff}}(\mathbf{R})} .
$$

The localization criterion can also be expressed by means of the conductivity measure [14]

Theorem 11 [14] The localization condition (17) is equivalent to

$$
\int_{\Delta \times \mathbf{R}} d m\left(E, E^{\prime}\right) \frac{1}{\left|E-E^{\prime}\right|^{2}}<\infty
$$

Let us define the Liouville operator $\mathcal{L}_{H}$ acting on $A \in \mathcal{A}$ by $\mathcal{L}_{H}(A)=\imath[H, A] / \hbar$. Then the spectral measure $\rho_{\vec{J}}$ of $\imath \mathcal{L}_{H}$ associated to the current operator $\vec{J}$ is defined by $\left(\right.$ for $f \in C_{0}(\mathbf{R})$ )

$$
\int d \rho_{\vec{J}}(\epsilon) f(\epsilon)=\frac{1}{d} \mathcal{T}\left(f\left(\imath \mathcal{L}_{H}\right)(\vec{J}) \cdot \vec{J}\right)
$$

Theorem 12 The spectral exponent $\alpha_{\rho_{\vec{J}}}(0)$ is given by

$$
\alpha_{\rho_{\vec{J}}}(0)=2\left(1-\sigma_{\mathrm{diff}}(\mathbf{R})\right)
$$




\subsection{Anomalous Drude Formula}

In [14], we showed that the zero frequency, isotropic direct conductivity at inverse temperature $\beta$, chemical potential $\mu$ and relaxation time $\tau_{\text {rel }}$ is given by

$$
\sigma_{\beta, \mu}=\frac{2 q^{2}}{\tau_{\text {rel }} \hbar^{2}} \int_{E \geq E^{\prime}} d m\left(E, E^{\prime}\right) \frac{f_{\beta, \mu}\left(E^{\prime}\right)-f_{\beta, \mu}(E)}{E-E^{\prime}} \frac{1}{\frac{1}{\tau_{\text {rel }}^{2}}+\left(\frac{E-E^{\prime}}{\hbar}\right)^{2}} .
$$

Here $f_{\beta, \mu}(E)$ is the Fermi-Dirac function $\left(1+e^{\beta(E-\mu)}\right)^{-1}, q$ is the particle charge and $\hbar$ is Planck's constant. More details on the derivation of (25) will be given in a forthcoming work.

Theorem 13 If $\beta<\infty$, the direct conductivity given in 25) satisfies

$$
\sigma_{\beta, \mu} \underset{\tau_{\text {rel }} \uparrow \infty}{\sim} \tau_{\text {rel }}^{-1+2 \sigma_{\text {diff }}(\mathbf{R})}
$$

If $\tau_{\text {rel }} \sim \beta^{\alpha}$ with $\alpha \approx 1-5$ as indicated in the introduction, a more detailed analysis shows that only exponents at the Fermi level $\mu$ intervene in the anomalous Drude formula.

\section{Exponents: generalities}

\subsection{Local regularity behavior}

In this section we compare different exponents characterizing the Hölder regularity behavior of positive functions. Although not all of these exponents will be used in this article, we present them for sake of completeness and later reference.

Definition 6 Let $f$ be a Lebesgue-measurable non-negative functions on the interval $(0, b]$, $b>0$. The exponents $\hat{\beta}$ and $\beta$ are defined by

$$
\hat{\beta}=\limsup _{x \rightarrow 0} \frac{\log f(x)}{\log x}, \quad \beta=\liminf _{x \rightarrow 0} \frac{\log f(x)}{\log x} .
$$

Remark 1 By convention a function vanishing in a neighborhood of the origin will have exponents equal to infinity.

Proposition 3 Let $f$ and $g$ be Lebesgue-measurable non-negative functions on the interval $(0, b], b>0$. Let $\alpha, \hat{\beta}$ and $\beta$ be as in Definitions 1 and 6 .

i) If $f(x) \underset{x \downarrow 0}{\sim} x^{\alpha}$, then $f(x) \log (x) \underset{x \downarrow 0}{\sim} x^{\alpha}$.

ii) (Calculation with Laplace transform) If $f(x) \underset{x \downarrow 0}{\sim} x^{\alpha}$ and $\alpha>-1$, then

$$
\int_{0}^{1} d t e^{-\delta t} f(t) \underset{\delta \uparrow \infty}{\sim} \delta^{-\alpha-1}
$$

iii) [40 The following equalities hold:

$$
\beta=\sup \left\{\gamma \in \mathbf{R} \mid \limsup _{x \downarrow 0} \frac{f(x)}{x^{\gamma}}<\infty\right\}, \quad \hat{\beta}=\inf \left\{\gamma \in \mathbf{R} \mid \liminf _{x \downarrow 0} \frac{x^{\gamma}}{f(x)}<\infty\right\}
$$


iv) $\beta \leq \alpha \leq \hat{\beta}$.

v) For $\alpha>0$ and $f$ non-decreasing (respectively, for $\alpha \leq 0$ and $f$ non-increasing), $\beta=\alpha$.

vi) Suppose that both $f$ and $g$ are non-increasing or non-decreasing with corresponding exponents $\alpha_{f}$ and $\alpha_{g}$ as defined in (91). Then for $\delta>0$,

$$
f(x)^{\delta} \underset{x \downarrow 0}{\sim} x^{\delta \alpha_{f}}, \quad f(x) g(x) \underset{x \downarrow 0}{\sim} x^{\alpha_{f}+\alpha_{g}}, \quad f(x)+g(x) \underset{x \downarrow 0}{\sim} x^{\min \left\{\alpha_{f}, \alpha_{g}\right\}} .
$$

Remark 2 These results transpose directly to the study of the behavior of a function at infinity as given in Definition 11. Note that in particular, if $g(x) \underset{x \uparrow \infty}{\sim} x^{\alpha}, \alpha>-1$, and $I(\delta)=$ $\int_{1}^{\infty} d x e^{-\delta x} g(x)$, then Proposition (3ii) and iii) show that

$$
\alpha=\inf \left\{\gamma \in \mathbf{R} \mid \limsup _{\delta \downarrow 0} \delta^{\gamma+1} I(\delta)=0\right\} .
$$

Remark 3 The following example will show that there exist functions with $\beta<\alpha<\hat{\beta}$ and for which the conclusions of Proposition $3 \mathrm{v}$ ) do not hold. Let $t>s>1, u \in \mathbf{R}$, and consider

$$
f(x)= \begin{cases}n^{u} & \text { for } x \in I_{n}=\left[\frac{1}{n^{s}}, \frac{1}{n^{s}}+\frac{1}{n^{s+t}}\right], \\ 0 & \text { otherwise }\end{cases}
$$

Because of (28) we have $\hat{\beta}=\infty$ and $\beta=-\frac{u}{s}$. By explicit calculation one gets

$$
\alpha=\frac{t-1}{s}-\frac{u}{s} .
$$

As an example, take $u=0, s=2$ and $t=5$, then $\beta=0, \alpha=2$ and $\hat{\beta}=\infty$. To consider the function $f^{\delta}$ is equivalent to replacing $u$ by $u \delta$ and this leads, according to (31), to a exponent different from $\delta \alpha$.

Proof of Proposition 3. i) This follows from the fact that $\int_{0}^{1} d x x^{-1+\epsilon} \log x<\infty$ for any $\epsilon>0$. ii) For $0>\gamma>-\alpha-1$, the identity

$$
\int_{1}^{\infty} \frac{d \delta}{\delta^{1+\gamma}} \int_{0}^{1} d t e^{-\delta t} f(t)=\int_{0}^{1} d t f(t) t^{\gamma} \int_{t}^{\infty} \frac{d s}{s^{1+\gamma}} e^{-s}
$$

allows to conclude.

iii) is proved in [40].

iv) Let us only show $\beta \leq \alpha$. The other inequality can be proved in a similar way. For any given $\delta>0$ there is a $\epsilon(\delta) \leq 1$ such that

$$
\inf _{x \leq \epsilon(\delta)} \frac{\log f(x)}{\log x}=\beta-\delta .
$$

Then for $x \leq \epsilon(\delta), f(x) \leq x^{\beta-\delta}$ because $\log x<0$. Let now $\gamma<\beta$ and choose $\delta$ such that $\beta-\delta-\gamma>0$, then

$$
\int_{0}^{\epsilon(\delta)} \frac{d x}{x} \frac{f(x)}{x^{\gamma}} \leq \int_{0}^{\epsilon(\delta)} \frac{d x}{x} x^{\beta-\delta-\gamma}<\infty
$$


which shows $\gamma \leq \alpha$.

v) We only treat the case where $\alpha>0$ and $f$ is non-decreasing. Take $0<\gamma<\alpha$, then if $x \leq a / 2$

$$
C(\gamma)=\int_{0}^{a} \frac{d y}{y} \frac{f(y)}{y^{\gamma}} \geq \int_{x}^{2 x} \frac{d y}{y} \frac{f(y)}{y^{\gamma}} \geq f(x) \int_{x}^{2 x} \frac{d y}{y^{1+\gamma}} \geq \frac{f(x)}{x^{\gamma}} \frac{1}{\gamma}\left(1-\frac{1}{2^{\gamma}}\right)
$$

and therefore equality (28) implies that $\gamma \leq \beta$ and hence $\alpha \leq \beta$. Thanks to iv) this gives $\alpha=\beta$.

$\mathbf{v i )}$ is a direct consequence of $\mathbf{i i i )}$ and $\mathbf{v}$ ).

\subsection{Local exponents and essential exponents}

We begin this section with the proof of Theorem 2. Then follow some comments on Definitions 2 and 3 and Theorem 2. In the rest of the section we prove the other results of Sections 2.3 and 2.4 as well as some complementary results.

The following lemma is known as the Hardy-Littlewood maximal inequality. We will need it in a slightly generalized form, nevertheless, its proof can be directly transposed from [67, for example.

Lemma 1 Let $\mu, \nu$ be two probability measures on $\mathbf{R}$ and $h \in L^{1}(\mathbf{R}, d \nu)$. The maximal function $M_{\mu, \nu, h}$ is defined by

$$
M_{\mu, \nu, h}(E)=\sup _{\epsilon \in(0,1]} \frac{1}{\mu([E-3 \epsilon, E+3 \epsilon])} \int_{(E-\epsilon, E+\epsilon)} d \nu\left(E^{\prime}\right) h\left(E^{\prime}\right)
$$

It is lower semicontinuous and satisfies for any positive $\lambda$ :

$$
\mu\left(\left\{E \in \mathbf{R} \mid M_{\mu, \nu, h}(E)>\lambda\right\}\right) \leq \frac{1}{\lambda}\|h\|_{L^{1}(\mathbf{R}, d \nu)} .
$$

Lemma 2 Let $\mu, \nu$ be two probability measures on $\mathbf{R}$. Then $\alpha_{\mu}(E) \leq \alpha_{\nu}(E) \mu$-almost surely.

Proof. If $M_{\mu, \nu, \mathbf{1}}(E)<\infty$, then $\nu((E-\epsilon, E+\epsilon))<C \mu([E-3 \epsilon, E+3 \epsilon])$ for all $\epsilon \in(0,1]$ and some constant $C>0$. Therefore $\alpha_{\mu}(E) \leq \alpha_{\nu}(E)$. Thus $\alpha_{\nu}(E)<\alpha_{\mu}(E)$ implies $M_{\mu, \nu, \mathbf{1}}(E)=\infty$. Hence by Lemma [1]

$$
\mu\left(\left\{E \in \mathbf{R} \mid \alpha_{\nu}(E)<\alpha_{\mu}(E)\right\}\right) \leq \mu\left(\bigcap_{N \in \mathbf{N}}\left\{E \in \mathbf{R} \mid M_{\mu, \nu, \mathbf{1}}(E)>N\right\}\right) \leq \lim _{N \rightarrow \infty} \frac{1}{N}=0
$$

Proof of Theorem 2. i) Clearly the local exponents are all bigger than or equal to 0. The exponents of the Lebesgue measure are all equal to 1. Applying Lemma 2 to the measure $\nu$ and the Lebesgue measure shows that $\alpha_{\nu}(E) \leq 1$ for $\nu$-almost all $E \in \mathbf{R}$.

ii) Apply Lemma 2 twice and use that $\mu$-almost surely implies $\nu$-almost surely.

iii) Since $\nu$ is pure-point, it is of the form $\sum_{n \in \mathbf{N}} c_{n} \delta\left(E-E_{n}\right), c_{n}>0$. For each $E_{n}$, $\nu\left(\left[E_{n}-\epsilon, E_{n}+\epsilon\right]\right) \geq c_{n}$ such that $\alpha_{\nu}\left(E_{n}\right)=0$. Consequently $\alpha_{\nu}(E)$ is equal to 0 for $\nu$-almost all $E$, notably the $E_{n}$ 's. 
iv) If $\nu$ is absolutely continuous, it is dominated by the Lebesgue measure. ii) allows to conclude.

v) We will prove a stronger result in Proposition điii) below.

Remark 4 Proposition 3 implies that the exponents $\alpha_{\nu}(E)$ are the same as those often considered in literature [81, 22, 53, 52, 48, 61, 6] because $\nu([E-\epsilon, E+\epsilon])$ is a non-decreasing function of $\epsilon$.

Remark 5 Theorem 2 does not exclude singular continuous spectrum with exponents equal to 0 or 1 .

Remark 6 An absolutely continuous measure can have exceptional points where the exponent is not equal to 1. For example, consider $d \nu(E)=h(E) d E \in \mathcal{M}$ with $h(E)=\left|E-E^{\prime}\right|^{\gamma}, \quad \gamma>-1$, on an interval around $E^{\prime}$. Then $\alpha_{\nu}\left(E^{\prime}\right)=1+\gamma$.

Remark 7 By definition $\gamma<\alpha_{\nu}^{-}(\Delta)$ if and only if there exists a set $\Xi \subset \Delta$ of zero $\nu$-measure such that $\gamma<\alpha_{\nu}(E)$ for all $E \in \Delta \backslash \Xi$. Furthermore $\gamma<\alpha_{\nu}^{+}(\Delta)$ if and only if there exists a set $\Xi \subset \Delta$ of stictly positive $\nu$-measure such that $\gamma<\alpha_{\nu}(E)$ for all $E \in \Xi$. Because the Borel function $E \mapsto \int_{0}^{1} \frac{d \epsilon}{\epsilon^{1+\gamma}} \int_{E-\epsilon}^{E+\epsilon} d \nu\left(E^{\prime}\right)$ is bounded on $\Xi$, Lusin's theorem then implies that there exists a set $\Xi^{\prime} \subset \Xi$ of positive $\nu$-measure such that $\int_{0}^{1} \frac{d \epsilon}{\epsilon^{1+\gamma}} \int_{E-\epsilon}^{E+\epsilon} d \nu\left(E^{\prime}\right)$ has a uniform bound for all $E \in \Xi^{\prime}$.

Definition 7 [74, 20, 48] The uniform dimension $\alpha_{\nu}^{\mathrm{uni}}(\Delta)$ of a measure $\nu$ on a Borel set $\Delta \subset \mathbf{R}$ is defined by

$$
\alpha_{\nu}^{\mathrm{uni}}(\Delta)=\sup \left\{\gamma \in \mathbf{R} \mid \exists C<\infty, \delta>0: \int_{E-\epsilon}^{E+\epsilon} \nu\left(d E^{\prime}\right) \leq C \epsilon^{\gamma} \forall \epsilon<\delta, E \in \Delta\right\}
$$

Remark 8 One clearly has $\alpha_{\nu}^{\text {uni }}(\Delta) \leq \alpha_{\nu}^{-}(\Delta)$. However, one does not necessarily have equality. For if

$$
f(E)=\sum_{q=2}^{\infty} \sum_{p=1}^{q-1} \frac{1}{q^{2}(q-1)} \frac{1}{|E-p / q|^{1 / 2}},
$$

then $f \in L^{1}([0,1])$ and defines an absolutely continuous probability measure $\nu=z^{-1} f d x$ (if $z>0$ is a normalization factor). Thus, for any Borel subset $\Delta$ of $[0,1], \alpha_{\nu}^{-}(\Delta)=1$ whereas if $\Delta$ contains some rational point, $\alpha_{\nu}^{\text {uni }}(\Delta) \leq 1 / 2$.

Now we present some further technical results as well as proofs of the other results of Sections 2.3 and 2.4 . 
Lemma 3 Let $N \in \mathbf{N}, \gamma>0$. If $\Delta \subset$ is a Borel set, then

$$
\mathcal{M}^{-}(\Delta, \gamma, N)=\left\{\nu \in \mathcal{M} \mid \int_{0}^{1} \frac{d \epsilon}{\epsilon^{1+\gamma}} \int_{E-\epsilon}^{E+\epsilon} d \nu(E) \leq N \text { for } \nu \text {-a.a. } E \in \Delta\right\}
$$

and

$$
\mathcal{M}^{+}(\Delta, \gamma, N)=\left\{\nu \in \mathcal{M} \mid \exists \Xi \subset \Delta, \nu(\Xi)>0, \int_{0}^{1} \frac{d \epsilon}{\epsilon^{1+\gamma}} \int_{E-\epsilon}^{E+\epsilon} d \nu\left(E^{\prime}\right) \leq N \text { for } E \in \Xi\right\}
$$

are Borel sets in $\mathcal{M}$. Furthermore,

$$
\mathcal{M}^{ \pm}(\Delta, \gamma, \infty)=\left\{\nu \in \mathcal{M} \mid \gamma<\alpha_{\nu}^{ \pm}(\Delta)\right\}
$$

are Borel sets.

Proof. Let $g_{k}(x)$ be a continuous non decreasing real function, equal to 0 for $x<0$, equal to 1 for $x>1 / k$ and $0 \leq g_{k}(x) \leq 1$ elsewhere. For $\chi \in C_{0}(\mathbf{R}), \delta>0, N \in \mathbf{N}$ and $\gamma>0$, the function

$$
\nu \in \mathcal{M} \rightarrow G_{k, \delta, \chi, N, \gamma}(\nu)=\int_{\mathbf{R}} d \nu(E) \chi(E) g_{k}\left(\int_{\delta}^{1} \frac{d \epsilon}{\epsilon^{1+\gamma}} \nu([E-\epsilon, E+\epsilon])-N\right) .
$$

is a continuous function. It is non-increasing in $\delta$. Since $\Delta$ is a Borel set, there exists a sequence $\chi_{n_{1}, m_{1}, \ldots, n_{r}, m_{r}} \in C_{0}(\mathbf{R})$, increasing in the $m_{j}$ and decreasing in the $n_{i}$, such that the characteristic function $\chi_{\Delta}$ is given by $\inf _{n_{1}} \sup _{m_{1}} \ldots \inf _{n_{r}} \sup _{m_{r}} \chi_{n_{1}, m_{1}, \ldots, n_{r}, m_{r}}$. Now

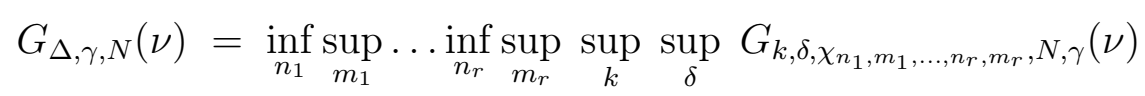

is a Borel function in $\nu$. By the dominated convergence theorem

$$
G_{\Delta, \gamma, N}(\nu)=\int_{\Delta} d \nu(E) g_{\infty}\left(\int_{0}^{1} \frac{d \epsilon}{\epsilon^{1+\gamma}} \nu([E-\epsilon, E+\epsilon])-N\right) .
$$

If $G_{\Delta, \gamma, N}(\nu)=0$ then there exists a set $\Xi$ of $\nu$-measure zero such that $\int_{0}^{1} \frac{d \epsilon}{\epsilon^{1+\gamma}} \nu([E-\epsilon, E+$ $\epsilon]) \leq N \quad \forall E \in \Delta \backslash \Xi$. Hence, $G_{\Delta, \gamma, N}(\nu)=0$ implies $\nu \in \mathcal{M}^{-}(\Delta, \gamma, N)$. Clearly $\nu \in$ $\mathcal{M}^{-}(\Delta, \gamma, N)$ implies $G_{\Delta, \gamma, N}(\nu)=0$. Consequently $\mathcal{M}^{-}(\Delta, \gamma, N)=G_{\Delta, \gamma, N}^{-1}(\{0\})$ is a Borel set. $\mathcal{M}^{+}(\Delta, \gamma, N)$ is treated in a similar way. Finally, the last result follows from $\mathcal{M}^{ \pm}(\Delta, \gamma, \infty)=$ $\bigcup_{N, M \in \mathbf{N}} \mathcal{M}^{ \pm}\left(\Delta, \gamma+\frac{1}{M}, N\right)$.

Proof of Proposition 1. Let $h$ denote the application $\nu \in \mathcal{M} \mapsto \alpha_{\nu}^{+}(\Delta)$. If $I=(a, b)$ is an open interval, it is sufficient to show that $h^{-1}(I)$ is a Borel set in order to deduce that $h$ is a Borel function. With the notations of Lemma 3 ,

$$
h^{-1}(I)=\mathcal{M}^{+}(\Delta, a, \infty) \cap\left(\bigcap_{n \in \mathcal{N}} \mathcal{M}^{+}\left(\Delta, b-\frac{1}{n}, \infty\right)\right)^{C},
$$

so that $h^{-1}(I)$ is a Borel set by Lemma 3 . The case of $\alpha_{\nu}^{-}(\Delta)$ is treated in a similar way.

Let $\mathcal{H}$ be a separable Hilbert space. We consider the space $\hat{\mathcal{M}}$ of $\mathcal{H}$-projection-valued Borel measures on $\mathbf{R}$ [57 endowed with the weak and vague topology, that is (because of the polarization identity) 


$$
\Pi_{n} \stackrel{\hat{\mathcal{M}}}{\rightarrow} \Pi \quad \Leftrightarrow \quad \int_{\mathbf{R}}\left\langle\phi\left|\Pi_{n}(d E)\right| \phi\right\rangle f(E) \rightarrow \int_{\mathbf{R}}\langle\phi|\Pi(d E)| \phi\rangle f(E),
$$

for all $\phi \in \mathcal{H}$ and $f$ in $C_{0}(\mathbf{R})$. To every self-adjoint operator $H$ the spectral theorem associates a $\Pi \in \hat{\mathcal{M}}$. Convergence in the strong resolvent sense corresponds to convergence in $\hat{\mathcal{M}}$.

Lemma 4 Let $\Pi \in \hat{\mathcal{M}}$ be a $\mathcal{H}$-projection valued Borel measure on a separable Hilbert space $\mathcal{H}$. Then there exists $\psi \in \mathcal{H}$ so that the spectral measure $\rho_{\psi}$ is in the same measure class as $\Pi$.

Proof. The lemma being well known, we only sketch an outline of the proof. A countable family of normalized vectors $\left(\phi_{i}\right)_{i \in I}$ is called $\Pi$-free if and only if $\int\left\langle\phi_{i}|\Pi(d E)| \phi_{j}\right\rangle f(E)=0$ for all $i \neq j$ and all $f \in C_{0}(\mathbf{R})$. The set of $\Pi$-free families is ordered by inclusion and Zorn's lemma assures the existence of a maximal family $\left(\phi_{i}\right)_{i \in I}$. Set

$$
\psi=c_{I} \sum_{n \in I} \frac{1}{2^{\frac{n+1}{2}}} \phi_{n}, \quad c_{I}=\frac{1}{\sqrt{1-2^{-\# I}}} .
$$

It is now possible to verify that the spectral measure $\rho_{\psi}$ of $\Pi$ dominates the spectral measure $\rho_{\eta}$ of any $\eta \in \mathcal{H}$.

Proof of Theorem [4. With Lemma t 1 choose $\phi \in \mathcal{H}$ such that $\rho_{\psi}$ is in the same measure class as $\Pi$. Then $\rho_{\psi}$ dominates the spectral measures $\rho_{\phi}$ for all $\phi \in \mathcal{H}$. Hence, by Theorem 2 , $\alpha_{\rho_{\phi}}^{+}(\Delta) \leq \alpha_{\rho_{\psi}}^{+}(\Delta) \leq \sup _{\eta \in \mathcal{H}} \alpha_{\rho_{\eta}}^{+}(\Delta)$ and $\alpha_{\rho_{\phi}}^{-}(\Delta) \geq \alpha_{\rho_{\psi}}^{-}(\Delta) \geq \inf _{\eta \in \mathcal{H}} \alpha_{\rho_{\eta}}^{-}(\Delta)$ for all $\phi \in \mathcal{H}$.

\section{Proposition 4 i)}

$$
\alpha_{\Pi}(E)=\sup \left\{\gamma \in \mathbf{R} \mid\left\|\int_{0}^{1} \frac{d \epsilon}{\epsilon^{1+\gamma}} \int_{E-\epsilon}^{E+\epsilon} \Pi\left(d E^{\prime}\right)\right\|_{\mathcal{B}(\mathcal{H})}<\infty\right\} .
$$

ii) Let $G_{\Pi}(z)=\int_{\mathbf{R}} \frac{\Pi\left(d E^{\prime}\right)}{z-E^{\prime}}$ be the resolvent of $\Pi$. Suppose $\alpha_{\Pi}(E) \in[0,2]$ then

$$
\left\|\int_{0}^{1} \frac{d \epsilon}{\epsilon^{1+\gamma}} \Im m\left(G_{\Pi}(E+\imath \epsilon)\right)\right\|_{\mathcal{B}(\mathcal{H})}<\infty,
$$

if and only if $\gamma<\alpha_{\Pi}(E)-1$.

iii) $(\Pi, E) \in \hat{\mathcal{M}} \times \mathbf{R} \rightarrow \alpha_{\Pi}(E)$ is a Borel function.

Proposition 5 Let $\mathcal{D}$ be a dense subset of $\mathcal{H}(\Delta)=\Pi(\Delta) \mathcal{H}$. Then

$$
\alpha_{\Pi}^{+}(\Delta)=\sup _{\phi \in \mathcal{D}} \alpha_{\rho_{\phi}}^{+}(\Delta), \quad \alpha_{\Pi}^{-}(\Delta)=\inf _{\phi \in \mathcal{D}} \alpha_{\rho_{\phi}}^{-}(\Delta) .
$$

The proof of the following lemma follows the lines of the proof of Lemma 3

Lemma 5 Let $N \in \mathbf{N}, \gamma>0$. The set

$$
\hat{\mathcal{S}}(\gamma, N)=\left\{(\Pi, E) \in \hat{\mathcal{M}} \times \mathbf{R} \mid \forall \phi \in \mathcal{H}: \int_{0}^{1} \frac{d \epsilon}{\epsilon^{1+\gamma}} \int_{E-\epsilon}^{E+\epsilon}\left\langle\phi\left|\Pi\left(d E^{\prime}\right)\right| \phi\right\rangle \leq N\right\},
$$

is closed in $\hat{\mathcal{M}} \times \mathbf{R}$. Furthermore, $\hat{\mathcal{S}}(\gamma, \infty)=\left\{(\Pi, E) \in \hat{\mathcal{M}} \times \mathbf{R} \mid \gamma<\alpha_{\Pi}(E)\right\}$ is a Borel set. 
Proof of Proposition 4. i) Let $\beta_{\Pi}(E)$ be the exponent on the right hand side of (32). Clearly $\beta_{\Pi}(E) \leq \alpha_{\Pi}(E)$. To show $\beta_{\Pi}(E) \geq \alpha_{\Pi}(E)$, let $\gamma<\alpha_{\Pi}(E)$. By the Schwarz inequality, the expression

$$
\left|\left\langle\psi\left|\int_{0}^{1} \frac{d \epsilon}{\epsilon^{1+\gamma}} \Pi([E-\epsilon, E+\epsilon])\right| \phi\right\rangle\right| \leq \sqrt{\int_{0}^{1} \frac{d \epsilon}{\epsilon^{1+\gamma}} \int_{E-\epsilon}^{E+\epsilon} d \rho_{\psi}\left(E^{\prime}\right)} \sqrt{\int_{0}^{1} \frac{d \epsilon}{\epsilon^{1+\gamma}} \int_{E-\epsilon}^{E+\epsilon} d \rho_{\phi}\left(E^{\prime}\right)}
$$

is bounded for all $\psi, \phi \in \mathcal{H}$. Consequently, the positive operator $\int_{0}^{1} \frac{d \epsilon}{\epsilon^{1+\gamma}} \Pi([E-\epsilon, E+\epsilon])$ is everywhere defined. By the Hellinger-Toeplitz theorem [57] it is therefore a bounded operator. Hence $\gamma<\beta_{\Pi}(E)$.

ii) This follows from Theorem 3 and an application of the Hellinger-Toeplitz theorem to

$$
\left\|\int_{0}^{1} \frac{d \epsilon}{\epsilon^{1+(\gamma-1)}} \Im m\left(G_{\Pi}(E-\imath \epsilon)\right)\right\|_{\mathcal{B}(\mathcal{H})}=\left\|\int_{0}^{1} \frac{d \epsilon}{\epsilon^{1+\gamma}} \int_{\mathbf{R}} \Pi\left(d E^{\prime}\right) \frac{\epsilon^{2}}{\left(E-E^{\prime}\right)^{2}+\epsilon^{2}}\right\|_{\mathcal{B}(\mathcal{H})}
$$

similar to i).

iii) Let $h$ be the application $(\Pi, E) \mapsto \alpha_{\Pi}(E)$. Let $I=(a, b)$ be an open interval. Then

$$
h^{-1}(I)=\hat{\mathcal{S}}(a, \infty) \cap\left(\bigcap_{n \in \mathbf{N}} \hat{\mathcal{S}}\left(b-\frac{1}{n}, \infty\right)\right)^{C},
$$

and Lemma 5 assures that $h^{-1}(I)$ is a Borel set. Therefore $h$ is a Borel function.

Proof of Proposition 5. Let us put $\beta=\sup _{\phi \in \mathcal{D}} \alpha_{\rho_{\phi}}^{+}(\Delta)$. Clearly $\beta \leq \alpha_{\Pi}^{+}(\Delta)$.

Let now $\psi \in \mathcal{H}$ be as in Theorem 4 and introduce $\Xi(\beta)=\left\{E \in \Delta \mid \alpha_{\rho_{\psi}}(E) \leq \beta\right\}$. As $E \mapsto \alpha_{\rho_{\psi}}(E)$ is a Borel function by Theorem 2, $\Xi(\beta)$ is a Borel set. Thus $\mathcal{H}(\beta)=\Pi(\Xi(\beta)) \mathcal{H}$ is a closed linear subspace of $\mathcal{H}(\Delta)$. Now for any $\phi \in \mathcal{D}, \rho_{\psi}$ dominates $\rho_{\phi}$ and therefore $\alpha_{\rho_{\phi}}(E)=\alpha_{\rho_{\psi}}(E) \rho_{\phi}$-almost surely by Theorem 2. Therefore

$$
\rho_{\phi}(\Delta) \geq \rho_{\phi}(\Xi(\beta)) \geq \rho_{\phi}\left(\left\{E \in \Delta \mid \alpha_{\rho_{\psi}}(E) \leq \alpha_{\rho_{\phi}}^{+}(\Delta)\right\}\right)=\rho_{\phi}(\Delta) .
$$

Hence $\rho_{\phi}(\Xi(\beta))=\|\Pi(\Delta) \phi\|^{2}=1$ and $\phi \in \mathcal{H}(\beta)$ for all $\phi \in \mathcal{D}$. Because $\mathcal{D}$ is dense in $\mathcal{H}(\Delta)$ by hypothesis, $\mathcal{H}(\Delta)=\mathcal{H}(\beta)$. Consequently, $\rho_{\psi}(\Xi(\beta))=\rho_{\psi}(\Delta)$ and $\alpha_{\rho_{\psi}}^{+}(\Delta) \leq \beta$. Theorem $\square$ implies $\alpha_{\Pi}^{+}(\Delta) \leq \beta$. This shows the first equality. In order to show the second equality, one proceeds in a similar way using the set of all $E \in \Delta$ such that $\beta \leq \alpha_{\rho_{\psi}}(E)$.

\subsection{Multifractal dimensions}

The formulæ on which the multifractal analysis developed below is based are already explicit in the article of Hentschel and Procaccia [36]. The dimensions introduced are often referred to as generalized Rényi dimensions [53, 52]. The main reason why this multifractal analysis is relevant for the quantum-mechanical study of solids is the following: the behavior of the Fourier transform of a measure at infinity which is of interest for physicists [38, 29, 40] can be rigorously linked to the 2 -spectral dimension of the measure, its correlation dimension. This will be done in the next section. Moreover, the multifractal dimensions give lower bounds on the lower essential dimension. Note that there are other possibilities to define multifractal dimensions 36, 35, 52, 53. 
Definition 8 Let $\nu \in \mathcal{M}$ and $\Delta \subset \mathbf{R}$ be a Borel set. If $\nu(\Delta) \neq 0$, let for $q \in \mathbf{R}$

$$
I_{\nu}^{q, \epsilon}(\Delta)=\lim _{p \downarrow q}\left(\int_{\Delta} \frac{d \nu(E)}{\nu(\Delta)}\left(\int_{E-\epsilon}^{E+\epsilon} d \nu\left(E^{\prime}\right)\right)^{p-1}\right)^{\frac{1}{p-1}}
$$

The q-spectral dimension $\alpha_{\nu}^{q}(\Delta)$ is defined by

$$
I_{\nu}^{q, \epsilon}(\Delta) \underset{\epsilon \downarrow 0}{\sim} \epsilon^{\alpha_{\nu}^{q}(\Delta)}
$$

unless $I_{\nu}^{q, \epsilon}(\Delta)$ is infinite for a set of $\epsilon$ 's of positive Lebesgue measure (possible if $q \leq 1$ ). We denote $\alpha_{\nu}^{q}=\alpha_{\nu}^{q}(\mathbf{R})$. The dimensions $\alpha_{\nu}^{1}$ and $\alpha_{\nu}^{2}$ are called information and correlation dimension respectively.

Remark 9 The notation is chosen such that in good cases the dimensions $\alpha_{\nu}^{q}$ are equal to the $D_{q}$ appearing in physics literature [36, 35, 29]. The dimensions $\alpha_{\nu}^{q}$ are rigorously linked to box-counting dimensions in [53, 52.

Remark 10 The limit in (34) is only introduced in order to study the case $q=1$. Using the monotone convergence theorem one gets

$$
I_{\nu}^{1, \epsilon}(\Delta)=\exp \left(\int_{\Delta} \frac{d \nu(E)}{\nu(\Delta)} \log \int_{E-\epsilon}^{E+\epsilon} d \nu\left(E^{\prime}\right)\right) .
$$

This explains why one talks of information dimension.

Proposition 6 Let $\nu \in \mathcal{M}$ and let $\Delta \subset \mathbf{R}$ be a Borel set.

i) For $q>1,0 \leq \alpha_{\nu}^{q}(\Delta) \leq \alpha_{\nu}^{-}(\Delta)$.

ii) 22] For $p \leq q, \alpha_{\nu}^{p}(\Delta) \geq \alpha_{\nu}^{q}(\Delta)$.

iii) $(q-1) \alpha_{\nu}^{q}(\Delta)$ is a convex function of $q$.

iv) $\alpha_{\nu}^{-}(\Delta) \leq \alpha_{\nu}^{1}(\Delta)$.

Proposition 7 Let $\nu \in \mathcal{M}$ and $\Delta \subset \mathbf{R}$ a Borel set.

i) If $\nu * \nu$ is the convolution of $\nu$ with itself, then $\alpha_{\nu * \nu}(0)=\alpha_{\nu}^{2}(\mathbf{R})$.

ii)

$$
\alpha_{\nu}^{2}(\Delta)=\sup \left\{\gamma \in \mathbf{R}\left|\int_{\Delta} d \nu(E) \int_{E-1}^{E+1} d \nu\left(E^{\prime}\right)\right| E-\left.E^{\prime}\right|^{-\gamma}<\infty\right\}
$$

iii) 61 The correlation dimension can be calculated as

$$
\int_{\mathbf{R}} d a\left|\Im m G_{\nu}(a+\imath \epsilon)\right|^{2} \underset{\epsilon \downarrow 0}{\sim} \epsilon^{\alpha_{\nu}^{2}-1}
$$


Remark 11 The multifractal dimensions are not measure class invariants. Let us give an example of an absolutely continuous measure for which the correlation dimension is smaller than 1:

$$
d \nu(E)=\operatorname{const} \frac{1}{E^{\beta}} e^{-E} \chi(E>0),
$$

where $\chi$ is the indicator function. It is a matter of calculation to verify that $\alpha_{\nu}^{2}=\min \{1,2(1-$ $\beta)\}$. This certainly limits their importance for a mathematical characterization of fractal measures.

Proof of Proposition 6. i) Since $q>1$, we get $I_{\nu}^{q, \epsilon}(\Delta) \leq 1$ for all $\epsilon>0$. Hence $\alpha_{\nu}^{q}(\Delta) \geq 0$.

Because $I_{\nu}^{q, \epsilon}(\Delta)$ is increasing in $\epsilon$, Proposition $3 \mathrm{v}$ ) and Fubini's theorem shows that

$$
(q-1) \alpha_{\nu}^{q}(\Delta)=\sup \left\{\gamma \in \mathbf{R} \mid \int_{\Delta} \frac{d \nu(E)}{\nu(\Delta)} \int_{0}^{1} \frac{d \epsilon}{\epsilon^{1+\gamma}}\left(\int_{E-\epsilon}^{E+\epsilon} d \nu\left(E^{\prime}\right)\right)^{q-1}<\infty\right\}
$$

Thus, for $\gamma<(q-1) \alpha_{\nu}^{q}(\Delta)$ and $\nu$-almost all $E \in \Delta, \int_{0}^{1} \frac{d \epsilon}{\epsilon^{1+\gamma}}\left(\int_{E-\epsilon}^{E+\epsilon} d \nu\left(E^{\prime}\right)\right)^{q-1}<\infty$. Using again the monotonicity in $\epsilon$ and Proposition $3 \mathrm{v}), \int_{0}^{1} \frac{d \epsilon}{\epsilon^{1+\gamma^{\prime}}} \int_{E-\epsilon}^{E+\epsilon} d \nu\left(E^{\prime}\right)<\infty$ for all $\gamma^{\prime}<\frac{\gamma}{q-1}$ and for $\nu$-almost all $E \in \Delta$, namely $\gamma^{\prime} \leq \alpha_{\nu}(E)$ for $\nu$-almost all $E \in \Delta$. Hence $\gamma^{\prime} \leq \alpha_{\nu}^{-}(\Delta)$ and therefore $\alpha_{\nu}^{q}(\Delta) \leq \alpha_{\nu}^{-}(\Delta)$.

ii) Let us show that for $q \neq 0, I_{\nu}^{q, \epsilon}(\Delta)$ is non-increasing functions of $\epsilon$ and a non-decreasing function in the variable $q$. This implies directly the result. Jensen's inequality for the convex function $f(t)=t^{\beta}, \beta \geq 1$ or $\beta \leq 0$, is used in the following way: let $p<1<q$ or $1<p<q$, then

$$
\int_{\Delta} \frac{d \nu(E)}{\nu(\Delta)}\left(\left(\int_{E-\epsilon}^{E+\epsilon} d \nu\left(E^{\prime}\right)\right)^{p-1}\right)^{\frac{q-1}{p-1}} \geq\left(\int_{\Delta} \frac{d \nu(E)}{\nu(\Delta)}\left(\int_{E-\epsilon}^{E+\epsilon} d \nu\left(E^{\prime}\right)\right)^{p-1}\right)^{\frac{q-1}{p-1}}
$$

that is, $I_{\nu}^{q, \epsilon}(\Delta) \leq I_{\nu}^{p, \epsilon}(\Delta)$. The case $p<q<1$ is treated in a similar way.

iii) Let $q=\sigma q_{0}+(1-\sigma) q_{1}$ with $\sigma \in[0,1]$. By Hölder's inequality one gets

$$
\left(I_{\nu}^{q, \epsilon}(\Delta)\right)^{q-1} \leq\left(I_{\nu}^{q_{0}, \epsilon}(\Delta)\right)^{\sigma\left(q_{0}-1\right)}\left(I_{\nu}^{q_{1}, \epsilon}(\Delta)\right)^{(1-\sigma)\left(q_{1}-1\right)} .
$$

Because the right hand side is increasing in $\epsilon$, Proposition $3 \mathrm{v}$ ) implies that it behaves as $\sigma\left(q_{0}-1\right) \alpha_{\nu}^{q_{0}}(\Delta)+(1-\sigma)\left(q_{1}-1\right) \alpha_{\nu}^{q_{1}}(\Delta)$. The exponent of the left hand side is $(q-1) \alpha_{\nu}^{q}(\Delta)$. The above inequality now implies that $(q-1) \alpha_{\nu}^{q}(\Delta) \geq \sigma\left(q_{0}-1\right) \alpha_{\nu}^{q_{0}}(\Delta)+(1-\sigma)\left(q_{1}-1\right) \alpha_{\nu}^{q_{1}}(\Delta)$.

iv) Because $I_{\nu}^{1, \epsilon}(\Delta)$ is monotone in $\epsilon$, Proposition $3 \mathrm{iv}$ ) and equation (35) imply that the exponent $\alpha_{\nu}^{1}(\Delta)$ is given by

$$
\alpha_{\nu}^{1}(\Delta)=\liminf _{\epsilon \rightarrow 0} \int_{\Delta} \frac{d \nu(E)}{\nu(\Delta)} \frac{\log \int_{E-\epsilon}^{E+\epsilon} d \nu\left(E^{\prime}\right)}{\log \epsilon} .
$$

By Fatou's lemma and again Proposition Biv), $\alpha_{\nu}^{1}(\Delta) \geq \nu-\operatorname{essinf}_{E \in \Delta} \alpha_{\nu}(E)$.

Proof of Proposition 7. i) By definition of the convolution

$$
\int_{E-\epsilon}^{E+\epsilon} d \nu * \nu\left(E^{\prime}\right)=\int_{\operatorname{diag}(E, \epsilon)} d \nu\left(E^{\prime}\right) d \nu\left(E^{\prime \prime}\right),
$$

where $\operatorname{diag}(E, \epsilon)=\left\{\left(E^{\prime}, E^{\prime \prime}\right)|| E-E^{\prime}+E^{\prime \prime} \mid<\epsilon\right\}$. But for $E=0$, this last expression is just equal to $I_{\nu}^{2, \epsilon}(\mathbf{R})$. Therefore $\alpha_{\nu * \nu}(0)=\alpha_{\nu}^{2}$. 
ii) follows by direct calculation using Fubini's theorem.

iii) Because of Theorem 3 and $\alpha_{\nu * \nu}(0)=\alpha_{\nu}^{2}(\mathbf{R})$, it is sufficient to show that

$$
\Im m G_{\nu * \nu}(-2 \imath \epsilon)=\frac{1}{\pi} \int_{\mathbf{R}} d a\left|\Im m G_{\nu}(a+\imath \epsilon)\right|^{2}
$$

For this purpose, write out the right hand side explicitly and use Fubini's theorem. The contour of the integral over $a$ can be closed by half of a circle in the upper half plane because the integrand falls off as $1 / a^{4}$ at infinity. There are two poles within the closed contour at $E+\imath \epsilon$ and $E^{\prime}+\imath \epsilon$. The residue theorem then allows to show (37).

\subsection{Asymptotic behavior of Fourier transforms}

In this section we study the asymptotic behavior of the Fourier transform of measures on the real line. It is governed by the correlation dimension of the measure. The first rigorous results in this direction were obtained by Strichartz [74]. He gave an estimate of the decrease of the Fourier transform by the uniform dimension of the measure (compare Theorem 15). Physicists interest began with the numerical works of [38] as well as 229. The latter work also contains a formal derivation of Theorem 14. Wavelet transform was used in a more mathematical approach in [40], further related results appear in [48, 6, 34]. Here, we present two versions of these results as well as an application to a quantitative version of the RAGE-theorem. These results are not new and we give them for sake of completeness.

The Fourier transform of $\nu$ is given by $\mathcal{F}_{\nu}(t)=\int d \nu(E) e^{\imath t E}$. Further let

$$
C_{\nu}(T)=\int_{0}^{T} \frac{d t}{T}\left|\mathcal{F}_{\nu}(t)\right|^{2} .
$$

Theorem 14 Let $\nu$ be a measure on the real line and $\alpha_{\nu}^{2}$ its correlation dimension. Then

$$
C_{\nu}(T) \underset{T \uparrow \infty}{\sim} T^{-\alpha_{\nu}^{2}} .
$$

The next corollary follows directly from Theorem 14, the Schwarz inequality and the equivalent of Proposition [3v) for the behavior at infinity.

Corollary 3 Let $\nu, \tilde{\nu}$ be two measures on the real line. If the functions $C_{\nu}(T)$ and $C_{\tilde{\nu}}(T)$ are eventually non-increasing, then

$$
\int_{0}^{T} \frac{d t}{T}\left|\mathcal{F}_{\nu}(t) \mathcal{F}_{\tilde{\nu}}(t)\right| \underset{T \uparrow \infty}{\sim} T^{-\alpha} \quad \text { with } \quad \alpha \geq \frac{1}{2}\left(\alpha_{\nu}^{2}+\alpha_{\tilde{\nu}}^{2}\right) \geq \min \left\{\alpha_{\nu}^{2}, \alpha_{\tilde{\nu}}^{2}\right\} .
$$

Moreover, if $\nu * \tilde{\nu}$ denotes the additive convolution, then one has $\alpha_{\nu * \tilde{\nu}}^{2} \geq\left(\alpha_{\nu}^{2}+\alpha_{\tilde{\nu}}^{2}\right)$.

Remark 12 It is certainly necessary to take the time average in equation (38). Consider, for example, an absolutely continuous measure $d \nu(E)=f(E) d E$ with a $C^{\infty}$ function $f$ of compact support. This implies that $\left|F_{\nu}(t)\right|^{2} \sim t^{-2 N}$ for any $N \in \mathbf{N}$ although the 2-spectral dimension of $\nu$ is $\alpha_{\nu}^{2}=1$. However, taking the time-average, one obtains $C_{\nu}(T) \sim T^{-1}$ in agreement with Theorem 14 . 
Theorem 15 [74] Let $\nu \in \mathcal{M}$. If $\nu$ has uniform dimension $\alpha_{\nu}^{\text {uni }}(\Delta)$ on a Borel set $\Delta \subset \mathbf{R}$ and $f \in L^{2}(\Delta, d \nu)$, then for any $\beta<\alpha_{\nu}^{\mathrm{uni}}(\Delta)$ and some positive constant $C$ :

$$
\int_{0}^{T} \frac{d t}{T}\left|\int_{\Delta} d \nu(E) f(E) e^{\imath E t}\right|^{2} \leq C\|f\|_{L^{2}(\Delta, d \nu)}^{2} T^{-\beta} .
$$

Before coming to the proofs, let us study the links between the diffusion of presence probability under quantum mechanical time evolution and spectral properties of the underlying Hamiltonian. This leads to a quantitative version of the RAGE-theorem [57].

Corollary 4 Let $\phi,\|\phi\|=1$, belong to a Hilbert space $\mathcal{H}$. Let $H$ a be self-adjoint operator on $\mathcal{H}$. Let $\rho_{\phi}$ be the spectral measure of $H$ associated to $\phi$. Then

$$
\int_{0}^{T} \frac{d t}{T}\left|\left\langle\phi\left|e^{-\imath H t}\right| \phi\right\rangle\right|^{2} \underset{T \uparrow \infty}{\sim} T^{-\alpha_{\rho_{\phi}}^{2}} .
$$

Remark 13 Let $\phi, \psi \in \mathcal{H},\|\phi\|=1,\|\psi\|=1$. If $\beta$ is defined by

$$
\int_{0}^{T} \frac{d t}{T}\left|\left\langle\phi\left|e^{-\imath H t}\right| \psi\right\rangle\right|^{2} \underset{T \uparrow \infty}{\sim} T^{-\beta}
$$

then

$$
\beta=\sup \left\{\gamma \in \mathbf{R}\left|\int_{\mathbf{R}} d \rho_{\phi, \psi}(E) \int_{\mathbf{R}} d \rho_{\psi, \phi}\left(E^{\prime}\right)\right| E-\left.E^{\prime}\right|^{-\gamma}<\infty\right\} .
$$

where $\rho_{\psi, \phi}$ is the complex spectral measure of $H$ associated to $\phi, \psi$. If either $\rho_{\phi}$ or $\rho_{\psi}$ has uniform dimension $\alpha^{\text {uni }}$, then one has $\beta \geq \alpha^{\text {uni }}$ as shows directly the spectral theorem and Theorem 15 .

Proof of Theorem 14. First note that $0 \leq \alpha_{\nu}^{2} \leq 1$. We have to show the equivalence between

$$
\int_{E \geq E^{\prime}} \frac{d \nu(E) d \nu\left(E^{\prime}\right)}{\left|E-E^{\prime}\right|^{\gamma}}<\infty
$$

and

$$
\int_{1}^{\infty} \frac{d T}{T^{1-\gamma}} \int_{E \geq E^{\prime}} d \nu(E) d \nu\left(E^{\prime}\right) \frac{2 \sin \left(\left(E-E^{\prime}\right) T\right)}{\left(E-E^{\prime}\right) T}<\infty,
$$

provided $0<\gamma<1$. Suppose that (42) holds. Because the integral

$$
\int_{1}^{\infty} \frac{d T}{T} \frac{\sin \left(\left|E-E^{\prime}\right| T\right)}{\left(\left|E-E^{\prime}\right| T\right)^{1-\gamma}}=\int_{\left|E-E^{\prime}\right|}^{\infty} d s \frac{\sin s}{s^{2-\gamma}}
$$

is absolutely convergent and behaves like $\mathcal{O}(1)$ as $\left|E-E^{\prime}\right| \rightarrow 0$, we may apply Fubini's theorem to

$$
\int_{E \geq E^{\prime}} \frac{d \nu(E) d \nu\left(E^{\prime}\right)}{\left|E-E^{\prime}\right|^{\gamma}} \int_{1}^{\infty} \frac{d T}{T} \frac{\sin \left(\left(E-E^{\prime}\right) T\right)}{\left(\left(E-E^{\prime}\right) T\right)^{1-\gamma}}<\infty
$$

in order to deduce (43). 
For the converse, let $N$ be any integer. By (43), there is a constant $C$ independent of $N$ such that

$$
C>\int_{1}^{N} \frac{d T}{T^{1-\gamma}} \int_{E-E^{\prime} \geq 0} d \nu(E) d \nu\left(E^{\prime}\right) \frac{\sin \left(\left(E-E^{\prime}\right) T\right)}{\left(E-E^{\prime}\right) T}>\int_{E-E^{\prime} \geq \frac{\pi}{N}} \frac{d \nu(E) d \nu\left(E^{\prime}\right)}{\left|E-E^{\prime}\right|^{\gamma}} \int_{E-E^{\prime}}^{N\left(E-E^{\prime}\right)} d s \frac{\sin s}{s^{2-\gamma}},
$$

because the integrand is positive as long as $E-E^{\prime}<\pi / N$. We have used Fubini's theorem. Then $0<\int_{0}^{\infty} d s \sin (s) / s^{2-\gamma}<\infty$ implies (42).

Proof of Theorem 15. Use the Cauchy-Schwarz inequality and $|\sin (\theta)| \leq|\theta|^{1-\beta}$ for all $\beta \in[0,1]$, to get :

$$
\int_{0}^{T} \frac{d t}{T}\left|\int_{\Delta} d \nu(E) f(E) e^{\imath E t}\right|^{2} \leq \int_{\Delta} d \nu(E)|f(E)|^{2} \int_{\Delta} d \nu\left(E^{\prime}\right)\left(\frac{2}{\left|E-E^{\prime}\right| T}\right)^{\beta} .
$$

Now if $\beta<\alpha_{\nu}^{\text {uni }}(\Delta)$, by definition there exists constants $C<\infty$ and $\delta>0$ such that $\nu((E-$ $\epsilon, E+\epsilon)) \leq C \epsilon^{\beta}$ for all $\epsilon \leq \delta$. Hence for $\beta<\beta^{\prime}<\alpha_{\nu}^{\text {uni }}(\Delta)$ one has (with changing constants $C)$ :

$$
\int_{\mathbf{R}} d \nu\left(E^{\prime}\right) \frac{1}{\left|E-E^{\prime}\right|^{\beta}} \leq \beta \int_{E-\delta}^{E+\delta} d \nu\left(E^{\prime}\right) \int_{\left|E-E^{\prime}\right|}^{\delta} \frac{d \epsilon}{\epsilon^{1+\beta}}+C \leq \beta \int_{0}^{\delta} \frac{d \epsilon}{\epsilon^{1+\beta}} C \epsilon^{\beta^{\prime}}+C \leq C .
$$

As this bound is uniform in $E$, this finishes the proof.

\section{Spectral exponents and anomalous quantum diffusion}

\subsection{Spectral exponents of covariant Hamiltonians}

Let $H=H^{*} \in \mathcal{A}$ be a given covariant Hamiltonian family. It gives rise to a covariant family $\left(\Pi_{\omega}\right)_{\omega \in \Omega}$ of projection-valued measures on $\mathcal{H}=\ell^{2}\left(\mathbf{Z}^{d}\right)$ by

$$
\int_{\mathbf{R}} \Pi_{\omega}(d E) f(E)=\pi_{\omega}(f(H)), \quad f \in C_{0}(\mathbf{R}) .
$$

For $\phi \in \mathcal{H}$, the corresponding spectral measure is denoted by $\rho_{\omega, \phi}$. Let us introduce the measure

$$
\rho_{\omega}=\sum_{n \in \mathbf{Z}^{d}} c_{n} \rho_{\omega,|n\rangle}
$$

where the sequence $\left(c_{n}\right)_{n \in \mathbf{Z}^{d}}$ of positive numbers satisfies $\sum_{n \in \mathbf{Z}^{d}} c_{n}=1$. By the following lemma, $\rho_{\omega}$ is in the same measure class as $\Pi_{\omega}$ and the application $\Pi_{\omega} \in \hat{\mathcal{M}} \mapsto \rho_{\omega} \in \mathcal{M}$ is continuous for fixed $\left(c_{n}\right)_{n \in \mathbf{Z}^{d}}$.

Lemma 6 Let $\left(\phi_{n}\right)_{n \in \mathbf{N}}$ be an orthonormal basis of $\mathcal{H}$. Let $\Pi \in \hat{\mathcal{M}}$ and introduce the measure $\rho_{\Pi}=\sum_{n=0}^{\infty} \frac{1}{2^{n+1}} \rho_{\phi_{n}}$. Then $\rho_{\Pi}$ is in the same measure class as $\Pi$ and the application $\Pi \in \hat{\mathcal{M}} \mapsto$ $\rho_{\Pi} \in \mathcal{M}$ is continuous.

Proof. If a Borel set $\Delta \subset \mathbf{R}$ satisfies $\rho_{\Pi}(\Delta)=0$, then $\rho_{\phi_{n}}(\Delta)=0$ for all $n \in \mathbf{N}$. Hence for $\psi=\sum_{n \in \mathbf{N}} a_{n} \phi_{n},\|\psi\|=1$, the Schwarz inequality gives $\rho_{\psi}(\Delta)=0$. Therefore $\rho_{\Pi}$ dominates $\Pi$. On the other hand, $\|\Pi(\Delta)\|=0$ clearly implies $\rho_{\Pi}(\Delta)=0$. Hence $\rho_{\Pi}$ and $\Pi$ are in the 
same measure class. Let now $\Pi_{l} \rightarrow \Pi$ in $\hat{\mathcal{M}}$ as $l \rightarrow \infty$. For $f \in C_{0}(\mathbf{R})$, by the dominated convergence theorem

$$
\lim _{l \rightarrow \infty} \int d \rho_{\Pi_{l}}(E) f(E)=\sum_{n \in \mathbf{N}} \frac{1}{2^{n+1}} \int\left\langle\phi_{n}|\Pi(d E)| \phi_{n}\right\rangle f(E)=\int d \rho_{\Pi}(E) f(E) .
$$

Therefore, $\rho_{\Pi_{l}} \rightarrow \rho_{\Pi}$ in $\mathcal{M}$ as $l \rightarrow \infty$.

Lemma 7 The applications $\omega \in \Omega \mapsto \Pi_{\omega} \in \hat{\mathcal{M}}$ and $\omega \in \Omega \mapsto \rho_{\omega} \in \mathcal{M}$ are continuous.

Proof. For $f \in C_{0}(\mathbf{R}), f(H) \in \mathcal{A}$ and the application $\omega \mapsto \pi_{\omega}(f(H))$ is strongly continuous. Because of equation (45) and the definition of the topology on $\hat{\mathcal{M}}$, this implies the continuity of the application $\omega \in \Omega \mapsto \Pi_{\omega} \in \hat{\mathcal{M}}$. The second statement now follows from Lemma 6 .

Proof of Theorem 5. We denote the spectral projection $\int_{\Delta} \Pi_{\omega}(d E)$ by $\Pi_{\omega}(\Delta)$ and introduce the function

$$
F_{\omega, E}(\gamma)=\left\|\int_{0}^{1} \frac{d \epsilon}{\epsilon^{1+\gamma}} \Pi_{\omega}([E-\epsilon, E+\epsilon])\right\| .
$$

The covariance implies that $F_{\omega, E}(\gamma)=F_{T^{-n} \omega, E}(\gamma)$ for all $n \in \mathbf{Z}^{d}$. The sets $\Omega_{\gamma, N}=\{\omega \in$ $\left.\Omega \mid F_{\omega, E}(\gamma) \leq N\right\}(n \in \mathbf{N} \cup\{\infty\})$ are therefore $T$-invariant. Moreover, $\Omega_{\gamma, N}=\left\{\omega \in \Omega \mid\left(\Pi_{\omega}, E\right) \in\right.$ $\hat{\mathcal{S}}(\gamma, N)\}$ is Borel (see Lemma 5). By the ergodicity of $\mathbf{P} \mathbf{P}\left(\Omega_{\gamma, N}\right)=0$ or 1 . The monotonicity of $F_{\omega, E}(\gamma)$ in $\gamma$ implies that, for $\gamma<\gamma^{\prime}, \Omega_{\gamma^{\prime}, \infty} \subset \Omega_{\gamma, \infty}$. Hence there exists a $\gamma_{c}$ such that for $\gamma<\gamma_{c}, \mathbf{P}\left(\Omega_{\gamma, \infty}\right)=1$, and for $\gamma>\gamma_{c}, \mathbf{P}\left(\Omega_{\gamma, \infty}\right)=0$. Because $\Omega_{\gamma, \infty}=\left\{\omega \in \Omega \mid \gamma<\alpha_{\Pi_{\omega}}(E)\right\}$, $\gamma_{c}=\alpha_{\Pi_{\omega}}(E) \mathbf{P}$-almost surely.

Let us now consider $\alpha_{\Pi_{\omega}}^{ \pm}(\Delta)$. The set $\Omega_{\gamma, \Delta}^{ \pm}=\left\{\omega \in \Omega \mid \gamma<\alpha_{\rho_{\omega}}^{ \pm}(\Delta)\right\}$ is $T$-invariant by Corollary 1 because $\rho_{\omega}$ and $\rho_{T^{a} \omega}$ are in the same measure class for any $a \in \mathbf{Z}^{d}$. Moreover, if $\mathcal{M}^{ \pm}(\gamma, \Delta, \infty)$ are the sets defined in Lemma 3, then $\Omega_{\gamma, \Delta}^{ \pm}=\left\{\omega \in \Omega \mid \rho_{\omega} \in \mathcal{M}^{ \pm}(\gamma, \Delta, \infty)\right\}$. The Lemma 3 and 7 imply that $\Omega_{\gamma, \Delta}^{ \pm}$are Borel sets. By ergodicity of $\mathbf{P}$, they have either full or zero P-measure. As $\gamma<\gamma^{\prime}$ implies $\Omega_{\gamma^{\prime}, \Delta}^{ \pm} \subset \Omega_{\gamma, \Delta}^{ \pm}$, there exist critical values $\gamma_{c}^{ \pm}$such that $\mathbf{P}\left(\Omega_{\gamma^{\prime}, \Delta}^{ \pm}\right)=1$ for $\gamma^{\prime}<\gamma_{c}^{ \pm}$and $\mathbf{P}\left(\Omega_{\gamma, \Delta}^{ \pm}\right)=0$ for $\gamma_{c}^{ \pm}<\gamma$.

Proof of Theorem 6. Let $\gamma<\alpha_{\text {LDOS }}(E)$. Then it follows from the arguments in the previous proof of Theorem 5 that there exists an $N<\infty$ such that the $T$-invariant set $\left\{\omega \in \Omega \mid\left(\Pi_{\omega}, E\right) \in\right.$ $\mathcal{S}(\gamma, N)\}$ has full measure. On this set of full measure the $F_{\omega, E}(\gamma)$ 's have uniform bound $N$, $\mathbf{P}$-almost surely. Therefore the spectral exponent satisfies

$$
\begin{aligned}
\alpha_{\mathrm{LDOS}}(E) & =\sup \left\{\gamma \in \mathbf{R} \mid \int_{\Omega} d \mathbf{P}(\omega) F_{\omega, E}(\gamma)<\infty\right\} \\
& \leq \sup \left\{\gamma \in \mathbf{R} \mid\left\|\int_{\Omega} d \mathbf{P}(\omega) \int_{0}^{1} \frac{d \epsilon}{\epsilon^{1+\gamma}} \int_{E-\epsilon}^{E+\epsilon} \Pi_{\omega}\left(d E^{\prime}\right)\right\|<\infty\right\}
\end{aligned}
$$

such that $\alpha_{\text {LDOS }}(E) \leq \inf _{\phi \in \mathcal{H}} \alpha_{\mathcal{N}_{\phi}}(E)$. Thus, $\alpha_{\text {LDOS }}(E) \leq \alpha_{\text {DOS }}(E)$.

Let now $\Omega_{1}=\left\{\omega \in \Omega \mid \alpha_{\rho_{\omega}}^{ \pm}(\Delta)=\alpha_{\mathrm{LDOS}}^{ \pm}(\Delta)\right\}$. If $g$ is the continuous application $\omega \in \Omega \mapsto$ $\rho_{\omega} \in \mathcal{M}$ (by Lemma 7) and $h$ the Borel function $\rho_{\omega} \in \mathcal{M} \mapsto \alpha_{\rho_{\omega}}^{ \pm}(\Delta)$ (by Proposition 1), then $\Omega_{1}=g^{-1}\left(h^{-1}\left(\left\{\alpha_{\mathrm{LDOS}}^{ \pm}(\Delta)\right\}\right)\right.$ is a Borel set. Moreover $\Omega_{1}$ is $T$-invariant and has full $\mathbf{P}$ measure.

Now let $I_{a}=\left\{(\omega, E) \in \Omega \times \mathbf{R} \mid \alpha_{\rho_{T^{a} \omega}}(E)=\alpha_{\rho_{\omega}}(E)\right\}$ for $a \in \mathbf{Z}^{d}$. If $k$ is the Borel function $(\omega, E) \in \Omega \times \mathbf{R} \mapsto \alpha_{\rho_{T^{a} \omega}}(E)-\alpha_{\rho_{\omega}}(E)$ (by Lemma 7 and Theorem 2aiv)), then $I_{a}=k^{-1}(\{0\}$ ) shows that $I_{a}$ is a Borel set. Hence $I=\bigcap_{a \in \mathbf{Z}^{d}} I_{a}$ is also a Borel set. Because $\rho_{\omega}$ and $\rho_{T^{a} \omega}$ are 
in the same measure class, Theorem 2 gives $\rho_{\omega}\left(\left\{E \in \mathbf{R} \mid(\omega, E) \in I_{a}\right\}\right)=1$ and by $\sigma$-additivity, $\rho_{\omega}(\{E \in \mathbf{R} \mid(\omega, E) \in I\})=1$.

Finally, let $\bar{\Delta}=\left\{(\omega, E) \in \Omega \times \Delta \mid \alpha_{\text {LDOS }}^{-}(\Delta) \leq \alpha_{\rho_{\omega}}(E) \leq \alpha_{\text {LDOS }}^{+}(\Delta)\right\}$. By Lemma 7 and Theorem 2 $2 \mathrm{iv}), \bar{\Delta}$ is a Borel set. It also satisfies $\rho_{\omega}(\{E \in \Delta \mid(\omega, E) \in \bar{\Delta}\})=\rho_{\omega}(\Delta)$ for $\mathbf{P}$-almost all $\omega \in \Omega$.

Now we set $\hat{\Delta}=I \cap\left(\Omega_{1} \times \mathbf{R}\right) \cap \bar{\Delta}$. It is a Borel set and $\rho_{\omega}(\{E \in \Delta \mid(\omega, E) \in \hat{\Delta}\})=\rho_{\omega}(\Delta)$ for $\mathbf{P}$-almost all $\omega \in \Omega$. If $(\omega, E) \in \hat{\Delta}$, then $\left(T^{a} \omega, E\right) \in \hat{\Delta}$ for all $a \in \mathbf{Z}^{d}$. If $\chi_{\hat{\Delta}}$ is the indicator function of $\hat{\Delta}$, then the definition of $\hat{\Delta}$ and Fubini's theorem give

$$
\int d \mathbf{P}(\omega) \int d \rho_{\omega}(E) \chi_{\hat{\Delta}}(\omega, E)=\mathcal{N}(\Delta)
$$

On the other hand, the invariance of $\mathbf{P}, \rho_{\omega,|n\rangle}=\rho_{T^{a} \omega,|n-a\rangle}$ and Fubini's theorem imply that

$$
\int d \mathbf{P}(\omega) \int d \rho_{\omega}(E) \chi_{\hat{\Delta}}(\omega, E)=\sum_{n \in \mathbf{N}} c_{n} \int d \mathbf{P}(\omega)\left(\frac{1}{|\Lambda|} \sum_{m \in \Lambda} \int d \rho_{\omega,|n-m\rangle}(E)\right) \chi_{\hat{\Delta}}(\omega, E) .
$$

for any $\Lambda \subset \mathbf{Z}^{d}$. By Birkhoff's theorem, in the limit of increasing rectangles centered at the origin $\Lambda \rightarrow \mathbf{Z}^{d}$, the term in the parenthesis converges to $\mathcal{N}, \mathbf{P}$-almost surely. Therefore, if we introduce the $T$-invariant Borel set $\Omega_{E}=\{\omega \in \Omega \mid(\omega, E) \in \hat{\Delta}\}$, Fubini's theorem gives

$$
\mathcal{N}(\Delta)=\int_{\Delta} d \mathcal{N}(E) \mathbf{P}\left(\Omega_{E}\right)
$$

Hence $\mathbf{P}\left(\Omega_{E}\right)=1$ for $\mathcal{N}$-almost all $E \in \Delta$. Consequently, because

$$
\Omega_{E}=\left\{\omega \in \Omega \mid \alpha_{\mathrm{LDOS}}^{-}(\Delta) \leq \alpha_{\rho_{\omega}}(E)=\alpha_{\rho_{T^{a}}}(E) \leq \alpha_{\mathrm{LDOS}}^{+}(\Delta) \forall a \in \mathbf{Z}^{d}\right\}
$$

by definition of $\hat{\Delta}, \alpha_{\text {LDOS }}^{-}(\Delta) \leq \alpha_{\text {LDOS }}(E) \leq \alpha_{\text {LDOS }}^{+}(\Delta)$ for $\mathcal{N}$-almost all $E \in \Delta$. Because $\alpha_{\mathrm{LDOS}}(E) \leq \alpha_{\mathrm{DOS}}(E), \alpha_{\mathrm{LDOS}}^{-}(\Delta) \leq \alpha_{\mathrm{DOS}}^{-}(\Delta)$ follows.

In order to show $\alpha_{\mathrm{LDOS}}^{+}(\Delta) \leq \alpha_{\mathrm{DOS}}^{+}(\Delta)$, it is now sufficient to show that

$$
\mathcal{N}-\operatorname{esssup}_{E \in \Delta} \alpha_{\text {LDOS }}(E)=\alpha_{\text {LDOS }}^{+}(\Delta)
$$

For this purpose, fix $\delta>0$ and introduce

$$
\Xi=I \cap\left(\Omega_{1} \times \mathbf{R}\right) \cap\left\{(\omega, E) \in \Omega \times \Delta \mid \alpha_{\mathrm{LDOS}}^{+}(\Delta)-\delta \leq \alpha_{\rho_{\omega}}(E) \leq \alpha_{\mathrm{LDOS}}^{+}(\Delta)\right\} .
$$

Then $\rho_{\omega}(\{E \in \mathbf{R} \mid(\omega, E) \in \Xi\})>0$, P-almost surely. Now introduce $\tilde{\Omega}_{E}=\{\omega \in \Omega \mid(\omega, E) \in \Xi\}$. Repeating the same arguments as above, there exists a set $\Xi_{\mathcal{N}}$ of positive $\mathcal{N}$-measure such that the $T$-invariant Borel set $\tilde{\Omega}_{E}$ has full $\mathbf{P}$-measure, that is for all $E \in \Xi_{\mathcal{N}}$,

$$
\alpha_{\mathrm{LDOS}}^{+}(\Delta)-\delta \leq \alpha_{\mathrm{LDOS}}(E) \leq \alpha_{\mathrm{LDOS}}^{+}(\Delta) .
$$

As $\delta>0$ is arbitrary, this finishes the proof.

Remark 14 Usually one expects averaged exponents to be smaller than exponents obtained by taking an essential infimum over disorder configurations. Actually, it is easy to check that $\alpha_{\mathrm{DOS}}(E) \leq \mathbf{P}-\operatorname{essinf}_{\omega \in \Omega} \alpha_{\rho_{\omega,|0\rangle}}(E)$. On the other hand, it is possible that the inequality 


$$
\mathbf{P}-\underset{\omega \in \Omega}{\operatorname{essinf}} \alpha_{\rho_{\omega,|0\rangle}^{-}}^{-}(\Delta)<\alpha_{\mathrm{DOS}}^{-}(\Delta)
$$

be realized. This is at the basis of Theorem 5. To understand the difficulty, let us consider a Hamiltonian with dense pure-point spectrum for $\mathbf{P}$-almost all $\omega \in \Omega$. Therefore $\mathbf{P}-\operatorname{essinf}_{\omega \in \Omega} \alpha_{\rho_{\omega,|0\rangle}}^{-}(\Delta)=0$. It is however well known that a given $E$ is $\mathbf{P}$-almost surely not in the spectrum so that $\alpha_{\mathrm{DOS}}(E)$ may be strictly bigger than 0 . The same may then hold for $\alpha_{\mathrm{DOS}}^{-}(\Delta)$.

As the exponents of the LDOS and the diffusion exponent, the exponents of the DOS do not depend on a given vector in Hilbert space as suggests the definition (12). For $\phi \in \mathcal{H}, \mathcal{N}_{\phi}$ is defined by

$$
\int d \mathcal{N}_{\phi}(E) f(E)=\int d \mathbf{P}(\omega)\left\langle\phi\left|\pi_{\omega}(f(H))\right| \phi\right\rangle, \quad f \in C_{0}(\mathbf{R}) .
$$

The DOS is then $\mathcal{N}=\mathcal{N}_{|0\rangle}$.

Proposition 8 For any $\phi \in \mathcal{H}$, the measure $\mathcal{N}_{\phi}$ is dominated by $\mathcal{N}$. If $E \in \mathbf{R}$ and $\Delta \subset \mathbf{R}$ a Borel set, then

$$
\alpha_{\mathrm{DOS}}(E)=\inf _{\phi \in \mathcal{H}} \alpha_{\mathcal{N}_{\phi}}(E), \quad \alpha_{\mathrm{DOS}}^{+}(\Delta)=\sup _{\phi \in \mathcal{H}} \alpha_{\mathcal{N}_{\phi}}^{+}(\Delta), \quad \alpha_{\mathrm{DOS}}^{-}(\Delta)=\inf _{\phi \in \mathcal{H}} \alpha_{\mathcal{N}_{\phi}}^{-}(\Delta)
$$

Proof. By Theorem 2 and Corollary [, it is sufficient to show that $\mathcal{N}$ dominates $\mathcal{N}_{\phi}$ for any $\phi \in \mathcal{H}$. Let $\Delta \subset \mathbf{R}$ be such that $\mathcal{N}(\Delta)=0$. If $\phi=\sum_{n \in \mathbf{Z}^{d}} \phi_{n}|n\rangle \in \mathcal{H}$, then $A=\sum_{n} \phi_{n} U(n) \in$ $L^{2}(\mathcal{A}, \mathcal{T})$ and

$$
\int d \mathcal{N}_{\phi}(E) f(E)=\mathcal{T}\left(f(H) A A^{*}\right)=\langle A|f(H)| A\rangle_{L^{2}(\mathcal{A}, \mathcal{T})} .
$$

Let $A_{n} \in \mathcal{A}$ be a Cauchy sequence in $L^{2}(\mathcal{A}, \mathcal{T})$ converging to $A$ such that $A_{n}$ be a trigonometric polynomial. Then $\left\langle A_{n}|f(H)| A_{n}\right\rangle$ converges to $\langle A|f(H)| A\rangle$ for any bounded $f(H)$. Since $A_{n}$ is a trigonometric polynomial, $\left\langle A_{n}\left|\chi_{\Delta}(H)\right| A_{n}\right\rangle=0$ where $\chi_{\Delta}$ is the characteristic function of the Borel set $\Delta$. Hence $\left\langle A\left|\chi_{\Delta}(H)\right| A\right\rangle=0$, that is $\mathcal{N}_{\phi}(\Delta)=0$.

\subsection{Diffusion exponents of covariant Hamiltonians}

Let us first generalize Definition .

Definition 9 Let $\delta X_{\omega, \Delta}^{2}(T)$ be the mean square displacement operator defined in (13). For $\phi \in \mathcal{H}$

$$
\int_{\Omega} d \mathbf{P}(\omega)\left\langle\phi\left|\delta X_{\omega, \Delta}^{2}(T)\right| \phi\right\rangle \underset{T \uparrow \infty}{\sim} T^{2 \sigma_{\phi}(\Delta)}
$$

and

$$
\left\langle\phi\left|\delta X_{\omega, \Delta}^{2}(T)\right| \phi\right\rangle \underset{T \uparrow \infty}{\sim} T^{2 \sigma_{\omega, \phi}(\Delta)}
$$

define the diffusion exponents $\sigma_{\phi}(\Delta)$ and $\sigma_{\omega, \phi}(\Delta)$. We set $\hat{\sigma}_{\phi}(\Delta)=\mathbf{P}-\operatorname{esssup}_{\omega \in \Omega} \sigma_{\omega, \phi}(\Delta)$ and $\sigma_{\text {diff }}(\Delta)=\sigma_{|0\rangle}(\Delta)$ where $|0\rangle$ is the state localized at the origin. 
Remark 15 Clearly $\hat{\sigma}_{\phi}(\Delta) \leq \sigma_{\phi}(\Delta)$. A strict inequality may be possible if $\left\langle\phi\left|\delta X_{\omega, \Delta}^{2}(T)\right| \phi\right\rangle$ has large fluctuations in $\omega$.

Proposition 9 i) For any $\phi \in \ell^{1}\left(\mathbf{Z}^{d}\right), \sigma_{\phi}(\Delta) \leq \sigma_{\text {diff }}(\Delta)$.

ii) If $\phi \in \mathcal{H}$ satisfies $\left\langle\phi\left|\Pi_{\omega}(\Delta) X^{2} \Pi_{\omega}(\Delta)\right| \phi\right\rangle<\infty$, then its diffusion exponent $\sigma_{\omega, \phi}(\Delta)$ can also be calculated by replacing the operator $\left(\vec{X}_{\omega}(t)-\vec{X}\right)^{2}$ in $(13)$ by $\vec{X}_{\omega}^{2}(t)$.

The rest of this section is devoted to proofs.

Proof of Proposition 2. By DuHamel's formula,

$$
\vec{\nabla}\left(e^{-\imath H t}\right)=-\imath \int_{0}^{t} d s e^{-\imath H(s-t)} \vec{\nabla}(H) e^{-\imath H s},
$$

where the integral is defined as a norm-convergent Riemann sum. Because $H \in \mathcal{C}^{1}(\mathcal{A}), \vec{\nabla}\left(e^{-\imath H t}\right)$ is an element of $\mathcal{A}$ and hence $\mathcal{T}\left(\Pi(\Delta)\left|\vec{\nabla}\left(e^{-\imath H t}\right)\right|^{2}\right)$ is well defined. Using the definition of the gradient, the cyclicity of the trace $\mathcal{T}$ and $\left[\Pi(\Delta), e^{-\imath H t}\right]=0$, one gets

$\mathcal{T}\left(\Pi(\Delta)\left|\vec{\nabla}\left(e^{-\imath H t}\right)\right|^{2}\right)=\mathcal{T}\left(\Pi(\Delta) e^{\imath H t}\left[\vec{X}, e^{-\imath H t}\right] \cdot\left[e^{\imath H t}, \vec{X}\right] e^{-\imath H t}\right)=\mathcal{T}\left(\Pi(\Delta)(\vec{X}(t)-\vec{X})^{2} \Pi(\Delta)\right)$

By definition of the trace $\mathcal{T}$ on $L^{\infty}(\mathcal{A}, \mathcal{T})$

$$
\int_{0}^{T} \frac{d t}{T} \mathcal{T}\left(\Pi(\Delta)(\vec{X}(t)-\vec{X})^{2} \Pi(\Delta)\right)=\int_{\Omega} d \mathbf{P}(\omega)\left\langle 0\left|\delta X_{\omega, \Delta}^{2}(T)\right| 0\right\rangle .
$$

This finishes the proof.

Proof of Theorem $7 \mathrm{i}$ ) and ii). i) $\sigma_{\text {diff }}(\Delta)$ is clearly bigger than or equal to 0 . Because $H \in \mathcal{C}^{1}(\mathcal{A})$, DuHamel's formula implies

$$
\left\|\vec{\nabla}\left(e^{\imath H t}\right)\right\| \leq \int_{0}^{t} d s\left\|e^{\imath H(t-s)} \vec{\nabla} H e^{\imath H s}\right\| \leq t\|\vec{\nabla}(H)\|
$$

so that

$$
\int_{0}^{T} \frac{d t}{T} \mathcal{T}\left(\Pi(\Delta)\left|\vec{\nabla}\left(e^{-\imath H t}\right)\right|^{2}\right) \leq \int_{0}^{T} \frac{d t}{T}\left\|\vec{\nabla}\left(e^{\imath H t}\right)\right\|^{2} \leq \frac{1}{3}\|\vec{\nabla}(H)\|^{2} T^{2}
$$

Hence the exponent $\sigma_{\text {diff }}(\Delta)$ is less than or equal to 1 .

ii) Let us use the algebra $\mathcal{A}_{\hat{H}, \hat{V}}$ common to $\hat{H}$ and $\hat{H}+\hat{V}$ introduced in the appendix. Then both Hamiltonians $H$ and $(H+V)$ are elements of $\mathcal{A}_{\hat{H}, \hat{V}}$ and hence so is $V=(H+V)-H$. Because $[\vec{X}, \hat{V}]$ is bounded, $V \in \mathcal{C}^{1}\left(\mathcal{A}_{\hat{H}, \hat{V}}\right)$. Therefore the diffusion exponent of $\hat{H}+\hat{V}$ is well defined by i). Because it is defined by means of the invariant ergodic measure $\mathbf{P}$, Theorem 1 implies the result.

Proof of Proposition 9. i) Let $\phi=\sum_{n \in \mathbf{Z}^{d}} \phi_{n}|n\rangle$ with $\sum_{n \in \mathbf{Z}^{d}}\left|\phi_{n}\right|<\infty$. By the Schwarz inequality

$$
\int_{\Omega} d \mathbf{P}(\omega)\left\langle\phi\left|\delta X_{\omega, \Delta}^{2}(T)\right| \phi\right\rangle \leq \int_{\Omega} d \mathbf{P}(\omega)\left\langle 0\left|\delta X_{\omega, \Delta}^{2}(T)\right| 0\right\rangle\|\phi\|_{\ell^{1}\left(\mathbf{Z}^{d}\right)}^{2}
$$

where we have used 


$$
\vec{X}_{\omega}(t)-\vec{X}=U(a)\left(\vec{X}_{T^{a} \omega}(t)-\vec{X}\right) U(a)^{*}
$$

and the invariance of the measure $\mathbf{P}$. Hence $\sigma_{\text {diff }}(\Delta) \geq \sigma_{\phi}(\Delta)$.

ii) Let $|\psi\rangle=\Pi_{\omega}(\Delta)|\phi\rangle$ and $\gamma>2 \sigma_{\phi}(\Delta) \geq 0$, then by Schwarz' inequality

$$
\left(\sqrt{\int_{1}^{\infty} \frac{d T}{T^{1+\gamma}} \int_{0}^{T} \frac{d t}{T}\left\langle\psi\left|\vec{X}_{\omega}(t)^{2}\right| \psi\right\rangle}-\sqrt{\int_{1}^{\infty} \frac{d T}{T^{1+\gamma}}\left\langle\psi\left|\vec{X}^{2}\right| \psi\right\rangle}\right)^{2}<\infty .
$$

Therefore the exponent defined by using only $\vec{X}_{\omega}^{2}(t)$ is necessarily smaller than or equal to $\sigma_{\phi}(\Delta)$. A similar argument shows the converse inequality, such that the exponents coincide.

Proof of Theorem 9. Using DuHamel's formula and basic properties of projections, one gets

$$
\int_{0}^{T} \frac{d t}{T} \mathcal{T}\left(\left|\vec{\nabla}\left(e^{-\imath H t}\right)\right|^{2} \Pi(\Delta)\right)=\int_{0}^{T} \frac{d t}{T} \int_{\Delta \times \mathbf{R}} d m\left(E, E^{\prime}\right) \frac{2-2 \cos \left(\left(E-E^{\prime}\right) t\right)}{\left(E-E^{\prime}\right)^{2}}
$$

Let now $\gamma \in \mathbf{R}$ be such that $2-\gamma>2 \sigma_{\text {diff }}(\Delta)$. Fubini's theorem then leads to

$$
\begin{gathered}
\int_{1}^{\infty} \frac{d T}{T^{1+(2-\gamma)}} \int_{0}^{T} \frac{d t}{T} \int_{\Delta \times \mathbf{R}} d m\left(E, E^{\prime}\right) \frac{2-2 \cos \left(\left(E-E^{\prime}\right) t\right)}{\left(E-E^{\prime}\right)^{2}} \\
\quad=2 \int_{\Delta \times \mathbf{R}} d m\left(E, E^{\prime}\right)\left|E-E^{\prime}\right|^{-\gamma} \int_{\left|E-E^{\prime}\right|}^{\infty} \frac{d s}{s^{1-\gamma}} \frac{s-\sin s}{s^{3}} .
\end{gathered}
$$

The integral over $s$ is bounded for $\gamma \in(0,2)$. Therefore, for $\sigma_{\text {diff }}(\Delta)<1$,

$$
2\left(1-\sigma_{\text {diff }}(\Delta)\right)=\sup \left\{\gamma \in \mathbf{R}\left|\int_{\Delta \times \mathbf{R}} d m\left(E, E^{\prime}\right)\right| E-\left.E^{\prime}\right|^{-\gamma}<\infty\right\},
$$

For $\sigma_{\text {diff }}(\Delta)=1$ this is immediately clear. The theorem now follows by direct calculation using Fubini's theorem.

Proof of Theorem 11. This follows from similar calculations as in the proof of Theorem 9 above.

Proof of Theorem 12. Let us consider $\imath \mathcal{L}_{H}$ as a self-adjoint operator on $L^{2}(\mathcal{A}, \mathcal{T})$. Then its spectral measure $\rho_{\vec{J}}$ associated to $\vec{J}=\vec{\nabla} H \in L^{2}(\mathcal{A}, \mathcal{T})$ is defined by (24) (the existence is guaranteed for by the Riesz-Markov theorem). It can be verified by direct calculation that for any polynomial $f$,

$$
\int d \rho_{\vec{J}}(\epsilon) f(\epsilon)=\int d m\left(E, E^{\prime}\right) f\left(E-E^{\prime}\right) .
$$

By density this extends to any $f \in C_{0}(\mathbf{R})$ such that the measures coincide. Using this in (48) for the case $\Delta=\mathbf{R}$ now allows to conclude. The second statement follows from the symmetry (20).

Proof of Theorem 10. Fubini's theorem and a contour integration in the upper half plane shows in the first place that

$$
2 \pi \imath \int_{\mathbf{R}} d a S_{m}(a+\imath \epsilon, a-\imath \epsilon)=\int_{\mathbf{R}^{2}} d m\left(E, E^{\prime}\right) \frac{1}{E-E^{\prime}-2 \imath \epsilon} .
$$


The latter is the Green's function $G_{\rho_{\vec{J}}}(2 \imath \epsilon)$ of the measure $\rho_{\vec{J}}$. By Theorem 3, $\Im m G_{\rho_{\vec{J}}}(2 \imath \epsilon) \underset{\epsilon \downarrow 0}{\sim}$ $\epsilon^{\alpha_{\rho_{\vec{J}}}(0)-1}$. On the other hand, $\alpha_{\rho_{\vec{J}}}(0)=2\left(1-\sigma_{\text {diff }}(\mathbf{R})\right)$ by equation (48) for $\Delta=\mathbf{R}$. The result follows.

Proof of Theorem 13. The integrand in (25) is clearly positive such that we may apply Fubini's theorem. After a change of variables we obtain

$$
\int_{1}^{\infty} \frac{d \tau_{\text {rel }}}{\tau_{\text {rel }}^{1+\gamma}} \sigma_{\beta, \mu}\left(\tau_{\text {rel }}, \hat{\omega}\right)=\frac{q^{2}}{\hbar} \int_{E \geq E^{\prime}} d m\left(E, E^{\prime}\right) \frac{f_{\beta, \mu}\left(E^{\prime}\right)-f_{\beta, \mu}(E)}{E-E^{\prime}}\left(\frac{E-E^{\prime}}{\hbar}\right)^{\gamma-1} \int_{\frac{\hbar}{E-E^{\prime}}}^{\infty} d s \frac{s^{\gamma}}{s^{2}-1} .
$$

The integral over $s$ is bounded for $-1<\gamma<1$. For $\beta<\infty$, the only singularity in the integrand of $m$ comes from the factor $\left(E-E^{\prime}\right)^{\gamma-1}$. The result now follows from Theorem 9 .

\subsection{The Guarneri bound}

The proof of the following result goes back to Guarneri [32], with considerable refinements due to [48, 6]. We refer the reader to [48, 6] for a proof.

Theorem Let $H$ be a Hamiltonian on $\mathcal{H}=\ell^{2}\left(\mathbf{Z}^{d}\right)$ and $\Pi \in \hat{\mathcal{M}}$ be its spectral family. Let $\Delta$ be a Borel spectral subset and $\phi \in \mathcal{H}$ satisfying $\left\langle\phi\left|\Pi(\Delta) X^{2} \Pi(\Delta)\right| \phi\right\rangle<\infty$. If $\rho_{\phi}$ is the spectral measure of $H$ associated to $\phi$ and $\sigma_{\phi}(\Delta)$ is defined by Definition 5 with $\Omega$ reduced to one point, then

$$
\alpha_{\rho_{\phi}}^{+}(\Delta) \leq d \cdot \sigma_{\phi}(\Delta)
$$

Remark 16 The result can be generalized to the study of other moments of the position operator:

$$
\int_{0}^{T} \frac{d t}{T}\left\langle\left.\phi|\Pi(\Delta)| X\right|^{\eta}(t) \Pi(\Delta) \mid \phi\right\rangle=\sum_{n \in \mathbf{Z}^{d}}|n|^{\eta} C_{T}(\phi, n, \Delta) \underset{T \uparrow \infty}{\sim} T^{\eta \cdot \sigma_{\phi}^{\eta}(\Delta)} .
$$

$\eta=2$ corresponds to the situation above. The inequality then reads $\alpha_{\rho_{\phi}}^{+}(\Delta) \leq d \cdot \sigma_{\phi}^{\eta}(\Delta)$. $\diamond$

Proof of Theorem $\nabla^{7 i i i) . ~ L e t ~} k$ be the smallest integer bigger than $d / 2$. Let $\phi \in \mathcal{D}\left(|\vec{X}|^{k}\right)$, the domain of the operator $|\vec{X}|^{k}$. If $k=2$, then the hypothesis $\left\|\pi_{\omega}\left(\vec{\nabla}^{2} H\right)\right\|<\infty$ and

$$
|\vec{X}|^{2} \pi_{\omega}(H)|\phi\rangle=\pi_{\omega}\left(\vec{\nabla}^{2} H\right)|\phi\rangle+2 \pi_{\omega}(\vec{\nabla} H) \cdot \vec{X}|\phi\rangle+\pi_{\omega}(H)|\vec{X}|^{2}|\phi\rangle
$$

imply that $\pi_{\omega}(H)$ leaves the domain $\mathcal{D}\left(|\vec{X}|^{2}\right)$ invariant. By functional calculus, $f\left(\pi_{\omega}(H)\right)$ also leaves $\mathcal{D}\left(|\vec{X}|^{2}\right)$ invariant for any $f \in C^{\infty}(\mathbf{R})$. Similar arguments treat the case of other $k$ 's. We set

$$
\mathcal{D}(\omega, \Delta)=\operatorname{span}\left\{f\left(\pi_{\omega}(H)\right)|n\rangle \mid n \in \mathbf{Z}^{d}, f \in C^{\infty}(\mathbf{R}), \operatorname{supp}(f) \subset \Delta\right\},
$$

where $\operatorname{supp}(f)$ denotes the support of $f$. As $\Delta$ is an open interval, $\mathcal{D}(\omega, \Delta)$ is dense in $\mathcal{H}(\omega, \Delta)=$ $\Pi_{\omega}(\Delta) \mathcal{H}$. Moreover, $\mathcal{D}(\omega, \Delta) \subset \mathcal{D}\left(|\vec{X}|^{k}\right)$ and 


$$
\|\phi\|_{\ell^{1}\left(\mathbf{Z}^{d}\right)} \leq\left(\sum_{n} \frac{1}{|n|^{2 k}}\right)^{\frac{1}{2}}\left(\sum_{n}|n|^{2 k}\left|\phi_{n}\right|^{2}\right)^{\frac{1}{2}}
$$

shows that $\mathcal{D}(\omega, \Delta) \subset \ell^{1}\left(\mathbf{Z}^{d}\right)$. Finally, let $\mathcal{D}^{\prime}$ be a countable subset of $\mathcal{D}(\omega, \Delta)$ still dense in $\mathcal{H}(\omega, \Delta)$.

Now for any $\phi \in \mathcal{D}^{\prime}$, Guarneri's inequality given above shows that $\alpha_{\rho_{\omega, \phi}}^{+}(\Delta) \leq d \cdot \sigma_{\omega, \phi}(\Delta)$. Thus

$$
\sup _{\phi \in \mathcal{D}^{\prime}} \alpha_{\rho_{\omega, \phi}}^{+}(\Delta) \leq d \sup _{\phi \in \mathcal{D}^{\prime}} \sigma_{\omega, \phi}(\Delta)
$$

Because of Proposition 5, the left hand side is equal to $\alpha_{\Pi_{\omega}}^{+}(\Delta)$ and by Theorem 5 to $\alpha_{\text {LDOS }}^{+}(\Delta)$. Recall that $\sigma_{\omega, \phi}(\Delta)$ is smaller than or equal to $\sigma_{\phi}(\Delta) \mathbf{P}$-almost surely. Because $\mathcal{D}^{\prime}$ is countable, there exists a set $\Omega_{1} \subset \Omega$ of full $\mathbf{P}$-measure, such that $\sigma_{\omega, \phi}(\Delta) \leq \sigma_{\phi}(\Delta)$ for all $\phi \in \mathcal{D}^{\prime}$ and $\omega \in \Omega_{1}$. Therefore $\mathcal{D}^{\prime} \subset \ell^{1}\left(\mathbf{Z}^{d}\right)$ gives

$$
\alpha_{\mathrm{LDOS}}^{+}(\Delta) \leq d \sup _{\phi \in \mathcal{D}^{\prime}} \sigma_{\phi}(\Delta) \leq d \sup _{\phi \in \ell^{1}\left(\mathbf{Z}^{d}\right)} \sigma_{\phi}(\Delta)
$$

But by Proposition 9ii) the right hand side is equal to $\sigma_{\text {diff }}(\Delta)$.

Proof of Theorem 8. i) and iii) are already proved in [14. (The definition of $l^{2}(\Delta)$ chosen in [14] was slightly different from (17) if, however, (17) is satisfied, it can be easily shown to be equivalent to the condition in [14.) ii) follows directly from the proof of of Theorem $7 \mathrm{ii}$ ).

\section{Example: Anderson model with free random variables}

As can be seen in equation (22), one needs to know the 2-particle Green function $G^{2}$ of a given model in order to calculate the corresponding conductivity measure. There are not many interesting models known in which $G^{2}$ can be calculated exactly. For Bloch electrons one can write out an explicit formula for the conductivity measure and then determine the diffusion exponent to be equal to 1 . On the other hand we already discussed the situation for models with localization in Section 4.3.

A class of solvable and non-trivial models has been studied by Wegner 80 and Khorunzhy and Pastur [47]. More recently, Neu and Speicher [51] considered a generalization of these models which will be the starting point here. The Hamiltonian in these models is given by the usual Anderson Hamiltonian $H=H_{0}+H_{1}$ where

$$
H_{0}=\sum_{r \neq s \in \mathbf{Z}^{d}} t_{|r-s|}|r\rangle\left\langle s\left|, \quad H_{1}=\sum_{r \in \mathbf{Z}^{d}} v_{r}\right| r\right\rangle\langle r|
$$

acting on $\ell^{2}\left(\mathbf{Z}^{d}\right)$, but the on-site disorder potentials $v_{r}$ are now supposed to be identically distributed free random variables in the sense of Voiculescu [79] instead of independent random variables. Random variables $X_{1}, X_{2}, \ldots$ are free if $\mathbf{E}\left(P_{1}\left(X_{r(1)}\right) P_{2}\left(X_{r(2)}\right) \cdots P_{m}\left(X_{r(m)}\right)\right)=0$ for any set of polynomials $P_{k}$ satisfying $\mathbf{E}\left(P_{k}\left(X_{r(k)}\right)\right)=0, k=1, \ldots, m$ whenever $r(k) \neq r(k+1)$, $k=1, \ldots, m-1$. We suppose that $t_{|n|} \leq|n|^{-d-1-\epsilon}$ for some $\epsilon>0$, so that $\partial_{j}(H)$ is bounded for any $j=1, \ldots, d$. As explained in [79, 51, Wegner's $n$-orbital model in the integrable limit $n \rightarrow \infty$ [80, the Anderson model in coherent potential approximation (CPA) and formally also the Lloyd model [49] are special cases of the Anderson model with free random variables. 
Strictly speaking this model is not covariant in the sense of Sections 2.1 and 2.2. The hull is here a non-commutative manifold. We have not studied the general formalism in detail hoping that the generalization to this case is indeed straightforward.

As a preamble, let us introduce the main tool for the addition of free random variables, notably Voiculescu's $R$-transform [79]. Given a Herglotz function $G(z)$ (namely, $G(z)$ satisfies $\Im m(G(z)) \Im m(z)<0)$, its $R$-transform is defined implicitly by the formula

$$
G(z)=\frac{1}{z-R(G(z))} .
$$

$R(G(z))$ has an analytic continuation to the upper half plane [51].

Examples: i) Wigner's semicircle law is given by the probability measure $\eta(\theta \in \mathbf{R})$ :

$$
d \eta(E)=\frac{1}{2 \pi \theta^{2}} \sqrt{4 \theta^{2}-E^{2}} \chi\left(E^{2} \leq 4 \theta^{2}\right) d E .
$$

Its Green's function and $R$-transform transform can be calculated explicitly:

$$
G(z)=\frac{z-\sqrt{z^{2}-4 \theta^{2}}}{2 \theta^{2}}, \quad R(z)=\theta^{2} z .
$$

Note that $\Im m(R(z)) \leq \theta^{2} \Im m(z)$ for $\Im m(z)<0$.

ii) Let $\eta$ be the uniform distribution on $[-1,1]$. Then its Green's function and $R$-transform transform are

$$
G(z)=\frac{1}{2} \log \frac{z-1}{z+1}, \quad R(z)=\frac{1}{\tanh z}-\frac{1}{z} .
$$

If $z=x-\imath y, y>0$, then

$$
\Im m(R(z))=\frac{\sin (2 y)}{\cos (2 y)-\cosh (2 x)}-\frac{y}{x^{2}+y^{2}} .
$$

Hence $R$ is not a Herglotz function in this example.

Let us first summarize the main results of [51]. The probability distribution of the $v_{r}$ 's is denoted by $\eta$. The space of disorder configurations $\Omega$ is a non-commutative measure space furnished with an expectation $\mathbf{E}$ which can be calculated by free convolution technics [79]. Transposing the notations of Sections 2.1 and 2.2 to the non-commutative hull $\Omega$, let $\pi_{\omega}(H)$ be the representation of the Hamiltonian corresponding to an element $\omega \in \Omega$. The following Green's functions are needed ( $\varsigma m z>0)$ :

$$
G_{0}(z)=\left\langle 0\left|\frac{1}{z-H_{0}}\right| 0\right\rangle, \quad G_{1}(z)=\int_{\mathbf{R}} d \eta\left(v_{r}\right) \frac{1}{z-v_{r}},
$$

and $G$ is the diagonal Green's function of the full Hamiltonian $H$, that is, of the DOS. Voiculescu's R-transforms [79 of these functions are denoted by $R_{0}$ and $R_{1}$. Finally, the non-diagonal Green's function $G(r-s, z)$ and its Fourier transform $\tilde{G}(q, z)$ are

$$
G(r-s, z)=\mathbf{E}_{\omega}\left(\left\langle r\left|\frac{1}{z-\pi_{\omega}(H)}\right| s\right\rangle\right)=\int_{\mathbf{B}} \frac{d^{d} q}{(2 \pi)^{d}} e^{\imath q \cdot(r-s)} \tilde{G}(q, z),
$$

where $\mathbf{B}=[-\pi, \pi)^{d}$ is the Brillouin zone. Let further $E_{0}(q)$ be the energy dispersion relation of $H_{0}$, that is the Fourier transform as in (50) of the function $t$ determining the kinetic Hamiltonian $H_{0}$. 
Theorem (Neu and Speicher [51]) Consider the Anderson model with free random variables described by the Hamiltonian (49) and suppose the support of the measure $\eta$ of the $v_{r}$ 's to be compact. Then Green's function satisfies the following equations

$$
G(z)=G_{0}\left(z-R_{1}(G(z))=G_{1}\left(z-R_{0}(G(z))\right.\right.
$$

and

$$
\tilde{G}(q, z)=\frac{1}{z-E_{0}(q)-R_{1}(G(z))} .
$$

Moreover, the 2-particle Green's function defined in (23) satisfies the identity

$$
\begin{aligned}
G^{2}\left(r, s, s^{\prime}, r^{\prime}, z_{1}, z_{2}\right)= & G\left(r-s, z_{1}\right) G\left(s^{\prime}, r^{\prime}, z_{2}\right)+\frac{R_{1}\left(G\left(z_{1}\right)\right)-R_{1}\left(G\left(z_{2}\right)\right)}{G\left(z_{1}\right)-G\left(z_{2}\right)} . \\
& \sum_{t \in \mathbf{Z}^{d}} G\left(r, t, z_{1}\right) G^{2}\left(t, s, s^{\prime}, t, z_{1}, z_{2}\right) G\left(t, r^{\prime}, z_{2}\right) .
\end{aligned}
$$

Finally, the solution of the usual Anderson model in CPA are also given by (51) and (53).

These results allow to calculate the diffusion exponent.

Theorem 16 The diffusion exponent $\sigma_{\text {diff }}=\sigma_{\text {diff }}(\mathbf{R})$ of the Anderson model with free random variables with compactly supported, absolutely continuous distribution is bigger than or equal to 1/2. The DOS is absolutely continuous. If moreover, $\Im m\left(R_{1}(z)\right) \leq C \Im m(z)$ for $\Im m(z)<0$ and $C \in \mathbf{R}_{+}$, then the diffusion exponent is equal to $1 / 2$. In particular, the diffusion exponent of the Wegner $n$-orbital model in the limit $n \rightarrow \infty$ is equal to $1 / 2$.

Proof of Theorem 16. According to a theorem of Voiculescu [78, a measure obtained by free convolution of an absolutely continuous measure with any other measure is absolutely continuous. The DOS is the free convolution of the spectral measures of $H_{0}$ and $H_{1}$ (see [51]), such that it is absolutely continuous because $H_{0}$ is so.

Let us now calculate the Stieltjes transform $S_{m}\left(z_{1}, z_{2}\right)$ of the conductivity measure of the free Anderson model. Because the kinetic part in (49) is symmetric, the matrix elements $\left\langle r\left|\vec{\nabla}\left(H_{0}\right)\right| s\right\rangle$ only depend on $r-s$ and therefore comparing with equation (22) shows that one actually needs to calculate the function

$$
\mathcal{G}^{2}\left(r, s, z_{1}, z_{2}\right)=\sum_{t \in \mathbf{Z}^{d}} G^{2}\left(r, t-s, t, 0, z_{1}, z_{2}\right)
$$

With the notations

$$
\mathcal{G}^{1}\left(r, z_{1}, z_{2}\right)=\sum_{t \in \mathbf{Z}^{d}} G\left(r-t, z_{1}\right) G\left(t, z_{2}\right), \quad \mathcal{R}\left(z_{1}, z_{2}\right)=\frac{R_{1}\left(G\left(z_{1}\right)\right)-R_{1}\left(G\left(z_{2}\right)\right)}{G\left(z_{1}\right)-G\left(z_{2}\right)},
$$

and using the fact that $G^{2}\left(r, s, s^{\prime}, r^{\prime}, z_{1}, z_{2}\right)$ only depends on three of its first four entries because the $v_{r}$ are identically distributed, summation of equation (53) leads to

$$
\mathcal{G}^{2}\left(r, s, z_{1}, z_{2}\right)=\mathcal{G}^{1}\left(r+s, z_{1}, z_{2}\right)+\mathcal{R}\left(z_{1}, z_{2}\right) \mathcal{G}^{2}\left(0, s, z_{1}, z_{2}\right) \mathcal{G}^{1}\left(r, z_{1}, z_{2}\right) .
$$

Putting $r=0$ and solving for $\mathcal{G}^{2}\left(0, s, z_{1}, z_{2}\right)$ one gets 


$$
\mathcal{G}^{2}\left(r, s, z_{1}, z_{2}\right)=\mathcal{G}^{1}\left(r+s, z_{1}, z_{2}\right)+\frac{\mathcal{R}\left(z_{1}, z_{2}\right) \mathcal{G}^{1}\left(r, z_{1}, z_{2}\right) \mathcal{G}^{1}\left(-s, z_{1}, z_{2}\right)}{1-\mathcal{R}\left(z_{1}, z_{2}\right) \mathcal{G}^{1}\left(0, z_{1}, z_{2}\right)}
$$

Remark that the quantity $\mathcal{G}^{2}$ only depends on the one-particle Green function in this model. Because $E_{0}(q)$ is an even function, so is $\tilde{G}(q, z)$ (cf. equation (52)) and then

$$
\mathcal{G}^{1}\left(r, z_{1}, z_{2}\right)=\int_{\mathbf{B}} \frac{d^{d} q}{(2 \pi)^{d}} e^{\imath q \cdot r} \tilde{G}\left(q, z_{1}\right) \tilde{G}\left(q, z_{2}\right)
$$

implies that $\mathcal{G}^{1}\left(r, z_{1}, z_{2}\right)$ is also an even function in its first variable. Now the matrix elements $\left\langle 0\left|\vec{\nabla}\left(H_{0}\right)\right| s\right\rangle$ change sign as the sign of $s$ is changed, and therefore the second term in (54) gives no contribution to the Stieltjes transform of the conductivity measure in (22). Passing to Fourier space the operator $\vec{\nabla}\left(H_{0}\right)$ becomes multiplication by the gradient of $E_{0}(q)$ with respect to quasi-moments and subsequent summation in (22) leads to the result

$$
S_{m}\left(z_{1}, z_{2}\right)=\int_{\mathbf{B}} \frac{d^{d} q}{(2 \pi)^{d}}\left|\vec{\nabla}_{q} E_{0}(q)\right|^{2} \frac{1}{z_{1}-E_{0}(q)-R_{1}\left(G\left(z_{1}\right)\right)} \frac{1}{z_{2}-E_{0}(q)-R_{1}\left(G\left(z_{2}\right)\right)} .
$$

For Wegner's $n$-orbital model, this equation has already been derived by Khorunzhy and Pastur [47]. Owing to Theorem 10 and equation (55), it is now necessary to consider the integral

$$
\int_{\mathbf{R}} d a \int_{\mathbf{B}} \frac{d^{d} q}{(2 \pi)^{d}}\left|\vec{\nabla}_{q} E_{0}(q)\right|^{2} \frac{1}{\left|a+\imath \epsilon-E_{0}(q)-R_{1}(G(a+\imath \epsilon))\right|^{2}},
$$

and to study its behavior in the limit $\epsilon \rightarrow 0$. Clearly the integral is strictly bigger than zero because of contributions for big $|a|$ and therefore the diffusion exponent is bigger than or equal to $1 / 2$ according to Theorem 10 .

In order to show that the above integral is bounded let us use its upper bound

$$
\begin{aligned}
\int_{\mathbf{R}} d a & \int_{\mathbf{B}} \frac{d^{d} q}{(2 \pi)^{d}} \frac{1}{\mid a+\imath \epsilon-E_{0}(q)-R_{1}\left(\left.G(a+\imath \epsilon)\right|^{2}\right.} \\
& =\int_{\mathbf{R}} d a \int_{\mathbf{R}} d \mathcal{N}_{0}(E) \frac{1}{\left|a+\imath \epsilon-E-R_{1}(G(a+\imath \epsilon))\right|^{2}} \\
& =\int_{\mathbf{R}} d a \frac{\Im m G(a+\imath \epsilon)}{\Im m\left(R_{1}(G(a+\imath \epsilon))\right)-\epsilon},
\end{aligned}
$$

where the last equality follows by direct calculation using the identity (51). The integrand in (56) is bounded by the hypothesis made on $R_{1}$ and it falls off as $1 / a^{2}$ at infinity. Therefore the integral is bounded and the diffusion exponent is equal to $1 / 2$.

In Wegner's $n$-orbital model the $v_{r}$ are given by $n \times n$ hermitian random matrices with independent Gaussian entries. The model then becomes exactly solvable in the limit $n \rightarrow \infty$. In fact, Voiculescu has shown that Gaussian random matrices are asymptotically free such that the $v_{r}$ in (49) are free in the limit $n \rightarrow \infty$. The distribution of the $v_{r}$ is then given by Wigner's semicircle law and thus the hypothesis on $R_{1}$ is satisfied ( $c f$. the above example).

Remark 17 Let us investigate the Lloyd model. The $v_{r}$ then follow the Cauchy distribution with parameter $\gamma>0$. Because all moments diverge, the calculations in [51] for the 2-point 
Green's function are only formal. Actually, the formal calculations lead to erroneous results as the following arguments show. One has $G_{1}(z)=1 /(z+\imath \gamma \operatorname{sign}(\Im m z))$ and this implies that $R_{1}(z)=\imath \gamma \operatorname{sign}(\Im m z)$. Use this for the calculation of $S_{m}$ in (55) which is supposed to hold. Because the Green's function is a Herglotz function, a contours integration leads to

$$
\Re e\left(\int_{\mathbf{R}} d a S_{m}(a+\imath \epsilon, a-\imath \epsilon)\right)=\frac{-1}{4 \pi} \frac{1}{\epsilon+\gamma} \int_{\mathbf{B}} \frac{d^{d} q}{(2 \pi)^{d}}\left|\vec{\nabla}_{q} E_{0}(q)\right|^{2} .
$$

According to Theorem 10 the diffusion exponent would therefore be equal to $1 / 2$. This would be independent of the dimension of the physical space and the strength of the coupling. In particular, it would imply regular diffusion in the one-dimensional Lloyd model. But this is in contradiction with the rigorous results of Aizenman and Molchanov [2] which imply that the diffusion exponent is equal to zero in this situation as is shown in [14].

It is possible, however, to calculate the exponent of the DOS of the Lloyd model using equation (51) and Theorem 3. These are then shown to be equal to 1 which is a well known result [23]. In fact, it is shown in [16] that the calculations in [51] are justified for the 1-point Green's function.

Remark 18 Formula (55) also holds for the case where $v_{r}$ 's are all equal to 0 . Then $R_{1}(z)=0$. Therefore a contour integration leads to

$$
\Re e\left(\int_{\mathbf{R}} d a S_{m}(a+\imath \epsilon, a-\imath \epsilon)\right)=\frac{1}{2 \pi \imath} \int_{\mathbf{B}} \frac{d^{d} q}{(2 \pi)^{d}}\left|\vec{\nabla}_{q} E_{0}(q)\right|^{2} \frac{1}{2 \imath \epsilon} \underset{\epsilon \downarrow 0}{\sim} \epsilon^{-1} .
$$

Comparison with Theorem 10 shows that $\sigma_{\text {diff }}(\mathbf{R})=1$ as expected for free particles.

Remark 19 The two other integrable models considered by Khorunzhy and Pastur [47 have a conductivity measure which is absolutely continuous with respect to the Lebesgue measure on $\mathbf{R}^{2}$ [47, eq. (2.29)]. For such an absolutely continuous conductivity measure, Theorem 9 implies directly a diffusion exponent $1 / 2$.

\section{Appendix}

Let us consider a topological dynamical system $\left(\Omega, T, \mathbf{Z}^{d}\right)$ where $\Omega$ is a compact metrisable space and $T$ is an action of $\mathbf{Z}^{d}$ by homeomorphisms. A point $\omega \in \Omega$ is called wandering whenever there is an open neighborhood $\mathcal{U}$ such that $T^{a}(\mathcal{U}) \cap \mathcal{U}=\emptyset$ for any $a \in \mathbf{Z}_{*}^{d}=\mathbf{Z}^{d} \backslash\{0\}$. The set $\Omega^{\mathrm{w}}$ of all wandering points is open. Hence its complement $\Omega^{\mathrm{nw}}$, the set of all non-wandering points, is compact.

Proposition 10 Any T-invariant probability $\mathbf{P}$ is supported on $\Omega^{\mathrm{nw}}$.

Proof. Let $\omega \in \Omega^{\mathrm{w}}$ and $\mathcal{U}$ be a neighborhood satisfying $T^{a}(\mathcal{U}) \cap \mathcal{U}=\emptyset$ for any $a \in \mathbf{Z}_{*}^{d}$. Then $T^{a}(\mathcal{U}) \cap T^{b}(\mathcal{U})=\emptyset$ for any $a \neq b \in \mathbf{Z}^{d}$. The $\sigma$-additivity and $T$-invariance of $\mathbf{P}$ imply

$$
\mathbf{P}(\Omega) \geq \mathbf{P}\left(\bigcup_{a \in \mathbf{Z}^{d}} T^{a}(\mathcal{U})\right)=\sum_{a \in \mathbf{Z}^{d}} \mathbf{P}(\mathcal{U})
$$


so that $\mathbf{P}(\mathcal{U})=0$. Therefore the support of $\mathbf{P}$ does not contain $\Omega^{\mathrm{w}}$.

Let us now consider the non-wandering points of the hull $\left(\Omega_{\hat{H}}, T, \mathbf{Z}^{d}\right)$ associated to a homogeneous Hamiltonian $\hat{H}$ on $\mathcal{H}=\ell^{2}\left(\mathbf{Z}^{d}\right)$ by (5). We denote $\hat{H} \in \Omega$ by $\omega_{0}$. The orbit of $\omega_{0}$ is the set $\operatorname{Orb}\left(\omega_{0}\right)=\left\{T^{a} \omega_{0} \mid a \in \mathbf{Z}^{d}\right\}$.

Lemma 8 Let us introduce the set

$$
\Omega_{\hat{H}}^{\infty}=\left\{\omega \in \Omega \mid \exists \text { sequence }\left(a_{n}\right)_{n \in \mathbf{N}} \text { in } \mathbf{Z}^{d}, \lim _{n \rightarrow \infty}\left|a_{n}\right|=\infty \text {, s.t. } \lim _{n \rightarrow \infty} T^{a_{n}} \omega_{0}=\omega\right\} \text {. }
$$

If $\omega_{0}$ is not an element of $\Omega_{\hat{H}}^{\infty}$, then $\Omega_{\hat{H}}^{\mathrm{w}}=\operatorname{Orb}\left(\omega_{0}\right)$. If $\omega_{0} \in \Omega_{\hat{H}}^{\infty}$, then $\Omega_{\hat{H}}^{\mathrm{w}}=\emptyset$. In both cases, $\Omega_{\hat{H}}^{\mathrm{nw}}=\Omega_{\hat{H}}^{\infty}$.

Proof. Let $\omega$ belong to $\Omega$. Then there exists a sequence $\left(a_{n}\right)_{n \in \mathbf{N}}, a_{n} \in \mathbf{Z}^{d}$, such that $\lim _{n \rightarrow \infty} T^{a_{n}} \omega_{0}=\omega$. Using the one-point compactification $\mathbf{Z}^{d} \cup\{\infty\}$ of $\mathbf{Z}^{d}$, one can extract a convergent subsequence $\left(a_{n_{k}}\right)_{k \in \mathbf{N}}$ with $\lim _{k \rightarrow \infty} T^{a_{n_{k}} \omega_{0}}=\omega$. If $\lim _{k \rightarrow \infty} a_{n_{k}}=\infty$, then $\omega \in \Omega_{\hat{H}}^{\infty}$, otherwise $\left(a_{n_{k}}\right)$ is a stationary sequence on some $a \in \mathbf{Z}^{d}$ and $\omega=T^{a} \omega_{0} \in \operatorname{Orb}\left(\omega_{0}\right)$. Hence $\Omega=\Omega_{\hat{H}}^{\infty} \cup \operatorname{Orb}\left(\omega_{0}\right)$, but this decompostion is not necessarily disjoint.

We next show that $\Omega_{\hat{H}}^{\infty} \subset \Omega_{\hat{H}}^{\text {nw }}$. For $\omega \in \Omega_{\hat{H}}^{\infty}$ there exists an unbounded sequence $\left(a_{n}\right)_{n \in \mathbf{N}}$ in $\mathbf{Z}^{d}$ such that $\lim _{n \rightarrow \infty} T^{a_{n}} \omega_{0}=\omega$. Hence for any neighborhood $\mathcal{U}$ of $\omega$ there is a $N \in \mathbf{N}$ such that $\left\{T^{a_{n}} \omega_{0} \mid n \geq N\right\} \subset \mathcal{U}$. Choose $a_{n} \neq a_{m}, n, m \geq N$. Then $T^{a_{n}} \omega_{0} \in \mathcal{U}$ and $T^{a_{m}-a_{n}}\left(T^{a_{n}} \omega_{0}\right) \in \mathcal{U}$ so that $T^{a_{m}-a_{n}}(\mathcal{U}) \cap \mathcal{U} \neq \emptyset$. As this holds for any neighborhood of $\omega, \omega \in \Omega_{\hat{H}}^{\text {nw }}$.

Now either $\omega_{0} \in \Omega_{\hat{H}}^{\mathrm{w}}$ or $\omega_{0} \in \Omega_{\hat{H}}^{\mathrm{nw}}$. In the first case, $\operatorname{Orb}\left(\omega_{0}\right) \subset \Omega_{\hat{H}}^{\mathrm{w}}$. According to the above, $\operatorname{Orb}\left(\omega_{0}\right)=\Omega_{\hat{H}}^{\mathrm{w}}$ and $\Omega_{\hat{H}}^{\mathrm{nw}}=\Omega_{\hat{H}}^{\infty}$. To deal with the second case, let us choose a metric on $\Omega$ compatible with the topology of $\Omega$. Open balls of radius $r$ around $\omega \in \Omega$ are denoted by $B(\omega, r)$. Because $\omega_{0} \in \Omega_{\hat{H}}^{\text {nw }}$, there exists for any $k \in \mathbf{N}$ an $a_{k} \in \mathbf{Z}_{*}^{d}$ such that $B\left(\omega_{0}, \frac{1}{k}\right) \cap T^{a_{k}} B\left(\omega_{0}, \frac{1}{k}\right) \neq \emptyset$. Therefore the sequence $T^{a_{k}} \omega_{0}$ converges to $\omega_{0}$. As $a_{k} \neq 0$ for all $k, \omega \in \Omega_{\hat{H}}^{\infty}$. Hence $\operatorname{Orb}\left(\omega_{0}\right) \subset$ $\Omega_{\hat{H}}^{\infty}$ and $\Omega_{\hat{H}}^{\mathrm{nw}}=\Omega_{\hat{H}}^{\infty}=\Omega$.

Proof of Theorem 1. Let $\hat{V}=\hat{V}^{*}$ be a compact operator of $\mathcal{H}=\ell^{2}\left(\mathbf{Z}^{d}\right)$. The hulls of the Hamiltonian $\hat{H}$ and the perturbed Hamiltonian $\hat{H}+\hat{V}$ are denoted by $\Omega_{\hat{H}}$ and $\Omega_{\hat{H}+\hat{V}}$. $\hat{V}$ compact implies that $\operatorname{sim}_{n \rightarrow \infty} U\left(a_{n}\right) \hat{V} U\left(a_{n}\right)^{*}=0$ for an unbounded sequence $\left|a_{n}\right| \rightarrow \infty$. Hence Lemma 8 implies $\Omega_{\hat{H}}^{\text {nw }}=\Omega_{\hat{H}+\hat{V}}^{\text {nw }}$. Proposition 10 and Lemma 8 show the other claims.

For a compact perturbation $\hat{V}$, let us introduce the common hull of $\hat{H}$ and $\hat{H}+\hat{V}$ by

$$
\Omega_{\hat{H}, \hat{V}}=\overline{\left\{\left(U(a) \hat{H} U(a)^{-1}, U(a) \hat{H}+\hat{V} U(a)^{-1}\right) \mid a \in \mathbf{Z}^{d}\right\}^{s}},
$$

where the closure is taken with respect to the strong topology on the Cartesian product $\mathcal{B}(\mathcal{H}) \times \mathcal{B}(\mathcal{H})$. By the arguments of the proof above, $\Omega_{\hat{H}, \hat{V}}^{\mathrm{nw}}=\left\{(\omega, \omega) \mid \omega \in \Omega_{\hat{H}}^{\mathrm{nw}}\right\}$. Hence $\left(\Omega_{\hat{H}, \hat{V}}^{\mathrm{nw}}, T, \mathbf{Z}^{d}\right)$ and $\left(\Omega_{\hat{H}}^{\mathrm{nw}}, T, \mathbf{Z}^{d}\right)$ are homeomorhpic as dynamical systems. Denoting by $p_{\hat{H}}\left(p_{\hat{H}+\hat{V}}\right)$ the projection from $\Omega_{\hat{H}, \hat{V}}$ to its first (second) component, we have the following picture:

$$
\begin{aligned}
& \Omega_{\hat{H}} \quad \overleftarrow{p_{\hat{H}}} \quad \Omega_{\hat{H}, \hat{V}} \quad \stackrel{p_{\hat{H}+\hat{V}}}{\longrightarrow} \Omega_{\hat{H}+\hat{V}} \\
& \Omega_{\hat{H}}^{\mathrm{nw}} \underset{{ }_{\hat{H}}}{\overleftrightarrow{\mathrm{H}}} \Omega_{\hat{H}, \hat{V}}^{\mathrm{nw}} \underset{{ }^{p_{\hat{H}+\hat{V}}}}{\overleftrightarrow{\mathrm{n}}} \Omega_{\hat{H}+\hat{V}}^{\mathrm{nw}}
\end{aligned}
$$

To conclude, let us consider the crossed product $C^{*}$-algebras $\mathcal{A}_{\hat{H}}, \mathcal{A}_{\hat{H}+\hat{V}}$ and $\mathcal{A}_{\hat{H}, \hat{V}}$ constructed from the dynamical systems on $\Omega_{\hat{H}}, \Omega_{\hat{H}+\hat{V}}$ and $\Omega_{\hat{H}, \hat{V}}$ by the procedure of Section 
2.1. There is an injection $i_{\hat{H}}: \mathcal{A}_{\hat{H}} \hookrightarrow \mathcal{A}_{\hat{H}, \hat{V}}$ which sends $f \in C_{0}\left(\Omega_{\hat{H}} \times \mathbf{Z}^{d}\right)$ to a function $i_{\hat{H}}(f) \in C_{0}\left(\Omega_{\hat{H}, \hat{V}} \times \mathbf{Z}^{d}\right)$ independent of the second argument in $\Omega_{\hat{H}, \hat{V}}$. Similarly one has $i_{\hat{H}+\hat{V}}: \mathcal{A}_{\hat{H}+\hat{V}} \hookrightarrow \mathcal{A}_{\hat{H}, \hat{V}}$. On the other hand, because the dynamical systems $\left(\Omega_{\hat{H}, \hat{V}}^{\mathrm{nw}}, T, \mathbf{Z}^{d}\right)$ and $\left(\Omega_{\hat{H}}^{\text {nw }}, T, \mathbf{Z}^{d}\right)$ are homeomorphic, they only give rise to one crossed product $\mathcal{A}_{\hat{H}}^{\text {nw }}$. Let us introduce the ideal

$$
J_{\hat{H}}^{\mathrm{nw}}=\left\{A \in \mathcal{A}_{\hat{H}} \mid \pi_{\omega}(A)=0 \forall \omega \in \Omega_{\hat{H}}^{\mathrm{nw}}\right\},
$$

and similarly $J_{\hat{H}+\hat{V}}^{\text {nw }}$ and $J_{\hat{H}, \hat{V}}^{\text {nw }}$. Then $\mathcal{A}_{\hat{H}}^{\text {nw }}$ is the quotient of $\mathcal{A}_{\hat{H}}\left(\right.$ or $\mathcal{A}_{\hat{H}+\hat{V}}$ or $\mathcal{A}_{\hat{H}, \hat{V}}$ ) with respect to $J_{\hat{H}}^{\text {nw }}\left(\right.$ or $J_{\hat{H}+\hat{V}}^{\text {nw }}$ or $J_{\hat{H}, \hat{V}}^{\text {nw }}$ ). Hence by using the surjective applications on the quotient, one has

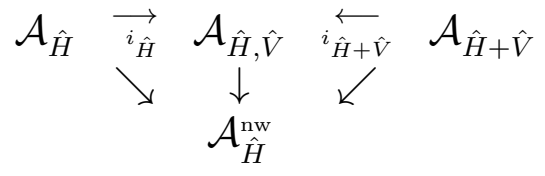

Let now a trace $\mathcal{T}$ be defined on $\mathcal{A}_{\hat{H}}\left(\right.$ or $\mathcal{A}_{\hat{H}+\hat{V}}$ or $\mathcal{A}_{\hat{H}, \hat{V}}$ ) by (8). As $\mathcal{T}$ vanishes on the ideals $J_{\hat{H}}^{\text {nw }}\left(\right.$ or $J_{\hat{H}+\hat{V}}^{\text {nw }}$ or $J_{\hat{H}, \hat{V}}^{\text {nw }}$ ) by Proposition [10, it is well defined on $\mathcal{A}_{\hat{H}}^{\text {nw }}$.

\section{References}

[1] E. Abrahams, P. W. Anderson, D. C. Licciardello and T.V. Ramakrishnan, Scaling Theory of Localization: Absence of Quantum Diffusion in Two Dimensions, Phys. Rev. Lett. 42, 673, (1979).

[2] M. Aizenman, S. Molchanov, Localization at Large Disorder and at Extreme Energies: an Elementary Derivation, Com. Math. Phys. 157, 245 (1993); and M. Aizenman, Localization at Weak Disorder: Some Elementary Bounds, Rev. Math. Phys. 6, 1163 (1994).

[3] R. Artuso, G. Casati, D. Shepelyansky, Fractal Spectrum and Anomalous Diffusion in the Kicked Harper Model, Phys. Rev. Lett. 68, 3826-3829 (1992).

[4] J. Avron, B. Simon, Almost Periodic Schrödinger Operators II. The Integrated Density of States Duke Math. J. 50, 369-391 (1983).

[5] N. W. Ashcroft, N. D. Mermin, Solid State Physics, (Saunders Co, Philadelphia, 1976).

[6] J.-M. Barbaroux, J.M. Combes, R. Montcho, Remarks on the relation between quantum dynamics and fractal spectra, to appear in J. Math. Ana. Appl.

[7] J. Bellissard, P. Moussa \& D. Bessis, Chaotic States of Almost Periodic Schrödinger Operators Phys. Rev. Lett. 49, 701-704 (1982).

[8] J. Bellissard, K-theory of $C^{*}$-algebras in solid state physics, in Statistical Mechanics and Field Theory: Mathematical Aspects, Lecture Notes in Physics 257, 99-156, edited by T. Dorlas, M. Hugenholtz, M. Winnink (Springer-Verlag, Berlin, 1986).

[9] J. Bellissard, B. Iochum, E. Scoppola, D. Testard, Spectral Properties of OneDimensional Quasicrystals, Comm. Math. Phys., 125, 527-543, (1989). 
[10] J. Bellissard, Spectral properties of Schrödinger operator with aThue-Morse potential, in Number Theory and Physics, J.M. Luck, P. Moussa \& M. Waldschmidt eds., 140-150, Springer Proceedings in Physics Vol.47, (Springer-Verlag, Berlin, 1990).

[11] J. Bellissard, A. Bovier, J.M. Ghez, Spectral Properties of a tight binding Hamiltonian with Period Doubling Potential, Comm. Math. Phys., 135, 379-399 (1991).

[12] J. Bellissard, Gap Labelling Theorems for Scrrödinger Operators, in From number theory to physics, edited by M. Waldschmidt, P. Moussa, J. Luck, C. Itzykson, 538-630 (Springer-Verlag, Berlin, 1991).

[13] J. Bellissard, Non Commutative Methods in Semiclassical Analysis, in Lecture Notes in Mathematics 1589, edited by S. Graffi, (Springer-Verlag, Berlin, 1994).

[14] J. Bellissard, A. van Elst, H. Schulz-Baldes, The Non-Commutative Geometry of the Quantum Hall Effect, J. Math. Physics 35, 5373 (1994).

[15] J. Bellissard, H. Schulz-Baldes, Anomalous transport in quasicrystals, in Proceedings of the 5th International Conference on Quasicrystals, Editors C. Janot, R. Mosseri, World Scientific, Singapore, 1995.

[16] H. Bercovici, D. Voiculescu, Free Convolution of Measures with unbounded support, Ind. U. Math. J. 42, 733-774 (1993).

[17] C. Berger, Electronic properties of quasicrystals experimental, in Lectures on quasicrystals, edited by F. Hippert, D. Gratias 463-504 (les éditions de physique, Les Ulis, 1994).

[18] A. Bovier, J.M. Ghez, Spectral properties of one-dimensional Schrödinger operators with potential generated by substitutions, Comm. Math. Phys., 158, 45-66 (1993).

[19] M. Casdagli, Symbolic dynamics for the renormalization map of a quasiperiodic Schrödinger equation, Comm. Math. Phys. 107, 295-318 (1986).

[20] J.-M. Combes, Connection between quantum dynamics and spectral properties of time evolution operators, in Differential Equations with Applications to Mathematical Physics, Ames W.F., Harell E.M., Herod J.V. Eds, Academic Press, Boston (1993).

[21] E. Contensou, $C^{*}$-algèbres des quasi-représentations d'un groupe discret et quasicristaux, $\mathrm{PhD}$ Thesis, Toulouse, 1996.

[22] C. Cutler, Some Results on the Behavior and Estimation of the Fractal Dimensions of Distributions on Attractors, J. Stat. Phy. 62 651-708 (1991).

[23] H. L. Cycon, R. G. Froese, W. Kirsch, B. Simon, Schrödinger operators with Applications to Quantum Mechanics and Global Geometry, (Springer-Verlag, New York, 1987)

[24] F. Delyon, D. Petritis, Recurrence of the eigenstates of a Schrödinger operator with automatic potential Comm. Math. Phys. 103, 441-444 (1986).

[25] F. Delyon, J. Peyrière, Recurrence of the eigenstates of a Schrödinger operator with automatic potential J. Stat. Phys. 64, 363-368 (1991). 
[26] W. Donoghue On the perturbation of spectra Comm. Pure Appl. Math., 18, 559-579 (1965).

[27] P. Drude, Zur Elektronentheorie der Metalle, Annalen der Physik 1, 566-613 (1900).

[28] K. Falconer, Fractal Geometry - Mathematical Foundations and Applications, (John Wiley \& Sons, 1990).

[29] T. Geisel, R. Ketzmerick, G. Peschel, New class of level statistics in quantum systems with unbounded diffusion, Phy. Rev. Lett. 66, 1651-1654 (1991).

[30] A. Gordon, A pure-point spectrum under 1-parameter perturbations and instability of Anderson localization, Comm. Math. Phys., 164, 489-505 (1994).

[31] D. Grempel, S. Fishman, R. Prange, Localization in an Incommensurable Potential: An Exactly Solvable Model, Phy. Rev. Lett. 49, 833-836 (1982).

[32] I. Guarneri, Spectral properties of quantum diffusion on discrete lattices, Europhys. Lett., 10, 95-100 (1989); and On an estimate concerning quantum diffusion in the presenc of a fractal spectrum, Europhys. Lett., 21, 729-733 (1993).

[33] I. Guarneri, G. Mantica, On the asymptotic properties of quantum dynamics in the presence of fractal spectrum, Ann. Inst H. Poincaré, 61, 369-379 (1994).

[34] C.-A. Guérin, M. Holschneider, Three equivalent definitions of the correlation dimension of a probability measure, preprint Marseille, 1995.

[35] T. Hasley, M. Jensen, L. Kadanoff, I. Procaccia, B. Shraiman, Fractal measures and their singularities: The characterization of strange sets, Phys. Rev A 33, 1141-1151 (1986).

[36] H. Hentschel, I. Procaccia, The infinite number of generalized dimensions of fractals and strange attractors, Physica 8D, 435-444 (1983).

[37] S. Hikami, A. I. Larkin, Y. Nagaoka, Prog. Theor. Phys., 63, 707, (1980).

[38] H. Hiramoto, S. Abe, Dynamics of an Electron in Quasiperiodic Systems. I. Fibonacci Model, J. Phys. Soc. Japan 57, 230-240 (1988); and Dynamics of an Electron in Quasiperiodic Systems. II. Harper Model, J. Phys. Soc. Japan 57, 1365-1372 (1988).

[39] A. Hof, O. Knill, B. Simon, Singular continuous spectrum for palindromic Schrödinger operator, to appear in Comm. Math. Phys..

[40] M. Holschneider, Fractal wavelet dimension and localization, Com. Math. Phys. 160, 457-474 (1994).

[41] J. S. Howland Perturbation Theory of dense Point Spectra J. Funct. Anal., 74, 52-80, (1987).

[42] C. Janot, Conductivity in quasicrystals via hierarchically variable-range hopping, Phys. Rev B 53, 181-191 (1996). 
[43] S. Jitomirskaya, Y. Last, Dimensional Hausdorff Properties of Singular Continuous Spectra, Phys. Rev. Lett. 76, 1765-1769 (1996).

[44] S. Jitomirskaya, B. Simon, Operators with singular continuous spectrum: III. Almost periodic Schrödinger operators, Comm. Math. Phys. 165, 201-205 (1994).

[45] J. Kellendonk Non commutative geometry of tilings and gap labelling. Rev. Math. Phys., 7, 1133-1180, (1995).

[46] M. Khomoto, Y. Oono, Cantor spectrum for an almost periodic Schödinger operator and a dynamical map, Phys. Lett. 102A, 145-148 (1984).

[47] A. Khorunzhy, L. Pastur, Limits of infinite interaction radius, dimensionality and the number of components for random operators with off-diagonal randomness, Com. Math. Phys. 153, 605-646 (1993).

[48] Y. Last, Quantum Dynamics and decomposition of singular continuous spectra, J. Funct. Ana. 142, 402-445 (1996).

[49] P. Lloyd, Exactly solvable model of electronic states in a three dimensional disordered Hamiltonian: non-existence of localized states, J. Phys. C 2, 1717-1725 (1969).

[50] D. Mayou, Introduction to the theory of electronic properties of quasicrystals, in Lectures on quasicrystals, edited by F. Hippert, D. Gratias, 417-462 (les éditions de physique, Les Ulis, 1994).

[51] P. Neu, R. Speicher, Rigorous mean field model for CPA: Anderson model with free random variables, J. Stat. Phys. 80, 1279-1308 (1995).

[52] L. Olsen, A Multifractal Formalism, Adv. in Mathematics, (1995).

[53] Y. Pesin, On Rigorous Mathematical Definitions of Correlation Dimension and Generalized Spectrum for Dimensions, J. Stat. Phy. 71 529-547 (1993).

[54] F. Piéchon, M. Benakli, A. Jagannathan, Analytical results for Scaling Properties of the spectrum of the Fibonacci Chain, Phys. Rev. Lett. 74, 5248 (1995).

[55] F. Piéchon, Etude des propriétés électroniques d'hamiltoniens quasipériodiques: statistiques des spectres d'énergie et diffusion quantique, $\mathrm{PhD}$ thesis, Université de Paris-Sud, Centre Orsay, 1995.

[56] S.J. Poon, F. S. Pierce, Q. Guo, Science 261, 737, (1993).

[57] M. Reed, B. Simon, Methods of modern mathematical physics, Vol. I- IV (Academic Press, London, 1975).

[58] R. del Rio, N. Makarov, B. Simon, Operators with singular continuous spectrum: I. Rank-one operators, Comm. Math. Phys., 165, 59-67, (1994).

[59] R. del Rio, S. Jitomirskaya, N. Makarov, B. Simon, Singular continuous spectrum is generic, Bull. AMS 31, No 2, 208-212, (1994). 
[60] R. del Rio, S. Jitomirskaya, Y. Last, B. Simon, What is Localization ? Phys. Rev. Lett. 75, 117-119, (1995).

[61] R. del Rio, S. Jitomirskaya, Y. Last, B. Simon, Operators with singular continuous spectrum: IV. Hausdorff dimension, rank-one perturbations and localization, Preprint 1995, submitted to J. d'Analyse Math..

[62] S. Roche, Contribution à l'étude théorique du transport électronique dans les quasicristaux, PhD Thesis Grenoble, October 1996.

[63] S. Roche, D. Mayou, Conductivity of quasiperiodic systems: a numerical study, preprint, Grenoble, 1996.

[64] S. Roche, G. Trambly de la Laissardière, D. Mayou, Electronic transport properties of quasicrystals, J. Math. Phys. 38, 1794-1822 (1997).

[65] C. A. Rodgers, Hausdorff measures, (Cambridge University Press, Cambridge, 1970).

[66] M.A. Ruderman, C. Kittel, Indirect Exchange Coupling of Nuclear Magnetic Moments by Conduction Electrons, Phys. Rev. 96, 99-102 (1954); and T. Kasuya, Magnetic Properties of Cu-Mn Alloys, Phys. Rev. 106, 893-898 (1957).

[67] W. Rudin, Real and Complex Analysis, Third Edition, (McGraw-Hill International Edition, New York, 1987).

[68] B. Shklovskii, A. L. Effros, Electronic Properties of Doped Semiconductors, (SpringerVerlag, Berlin, 1984).

[69] B. Simon, Almost periodic Schrödinger operators: a review, Adv. Appl. Math., 3, 463490, (1982).

[70] B. Simon, Operators with singular continuous spectrum: I. General operators, Ann. Math., 141, 131-145, (1995).

[71] B. Simon, T. Wolff, Singular Continuous Spectrum under Rank One Perturbations and Localization for Random Hamiltonians, Comm. Pure Appl. Math. 39, 75-90 (1986).

[72] C. Sire, D. Gratias, Introduction to the physics of quasicrystals, in NATO ASI Series B: Physics Vol. 319, 127-154, edited by P. Turchi and A. Gonis, (Plenum Press, New York and London, 1994).

[73] C. Sire, B. Passaro, V. Benza, Anomalous diffusion and conductivity in octagonal tiling models, Phys. Rev., B46, 13751-13754, (1992); and Electronic properties of 2d quasicrystals. Level spacing distribution and diffusion, J. Non Crystalline Sol., 153 \&154, 420-425, (1993).

[74] R.S. Strichartz, Fourier Asymptotics of Fractal Measures, J. Funct. Ana. 89, 154-187 (1990).

[75] A. Sütó, The spectrum of a quasiperiodic Schrödinger operator, Com. Math. Phys. 111, 409-415 (1987). 
[76] A. Sütó, Singular Continuous Spectrum on a Cantor Set of Zero Lebesgue Measure for the Fibonacci Hamiltonian, J. Stat. Phys. 56, 525-530 (1989).

[77] D. J. Thouless, Maximum Metallic Resistance in Thin Wires, Phys. Rev. Lett. 39, 1167-1169 (1977).

[78] D. Voiculescu, The Analogues of Entropy and of Fisher's Information Measure in Free Probability Theory, Com. Math. Phys. 155, 71-93 (1993).

[79] D. Voiculescu, K. Dykema, A. Nica, Free random variables, (AMS, Providence, Rhode Island, 1992).

[80] F. Wegner, Disordered system with $n$ orbitals per site: $n=\infty$ limit, Phys. Rev B 19, 783-792 (1979).

[81] L.-S. Young, Dimension, entropy and Lyapunov exponents, Erg. Th. Dyn. Sys. 2, 109124 (1982).

[82] J. X. Zhong, J. Bellissard, R. Mosseri, Green function analysis of energy spectra scaling properties, J. Phys: Condens. Matter 7, 3507-3514 (1995). 